\title{
The role of excited-state character, structural relaxation, and sym- metry breaking in enabling delayed fluorescence activity in push-pull chromophores
}

\author{
Patrick Kimber, ${ }^{a}$ Pooja Goddard, ${ }^{a}$ lain A. Wright, ${ }^{a}$ Felix Plasser ${ }^{* a}$
}

Received Date Accepted Date

DOI: $00.0000 / x x x x x x x x x x$
Thermally activated delayed fluorescence (TADF) is a current promising route for generating highly efficient light-emitting devices. However, the design process of new chromophores is hampered by the complicated underlying photophysics that requires a number of different pathways to be optimised simultaneously. In this work, four closely related donor- $\pi$-acceptor- $\pi$-donor systems have been investigated, two of which were synthesised previously, with the aim of elucidating their varying effectiveness for TADF. We, first, outline that neither the frontier orbitals nor the singlet-triplet gaps are sufficient in discriminating between the molecules. Subsequently, a detailed analysis of the excited states, performed at a correlated ab initio level, is shown highlighting the presence of a number of closely spaced singlet and triplet states of varying character. Five density functionals are benchmarked against this reference revealing dramatic changes in, both, excited state energies and wavefunctions following variations in the amount of Hartree-Fock exchange included. Excited-state minima are optimised in solution showing the crucial role of structural variations for stabilising locally excited and CT states and of symmetry breaking for producing a strongly emissive $S_{1}$ state. More generally, this work shows how a detailed analysis of excited-state wavefunctions can provide critical new insight into excited-state electronic structure, helping to reveal the photophysics of existing push-pull chromophores and ultimately guiding the design of new ones.

\section{Introduction}

Thermally activated delayed fluorescence (TADF) constitutes a promising route toward creating organic electroluminescent materials with the potential to obtain $100 \%$ internal quantum efficiency through the possibility of harvesting both singlet and triplet excitons. 1 - 3 on a microscopic level, TADF is governed by the reverse intersystem crossing (rISC) process whereby a nonemissive molecular triplet excited state is converted into an emissive singlet state capable of efficient luminescence. Most prominently, the rISC process depends on the energy gap $\left(\Delta E_{S T}\right)$ between the lowest excited singlet and triplet states, which enters exponentially into the rate expression ${ }^{1}$ and can be seen as an effective activation energy to the TADF process. Other influences are related to spin-orbit coupling (SOC), enabling the formally spin-forbidden rISC process, 1 and the oscillator strength $(f)$ of the emitting singlet state. ${ }^{4}$ All three properties crucially depend on the state character where enhanced charge transfer (CT) is expected to lower both $\Delta E_{S T}$ and $f$. SOC, on the other hand, is promoted by a difference in state character between the singlet and triplet states. 15.7 More recently, multipolar systems have been introduced where one acceptor unit is connected to two or more donor units and vice versa. $\frac{48}{8}$ Aside from the above considerations, the possibility of symmetry breaking in the excited state is particularly intriguing in such multipolar systems. .89

\footnotetext{
${ }^{a}$ Department of Chemistry, Loughborough University, Loughborough, LE11 3TU, U.K.; Tel: +44 1509 226946; E-mail: f.plasser@lboro.ac.uk

$\dagger$ Electronic Supplementary Information (ESI) available: relative energies of con-
} formers, fragment definitions, and analysis of vertical excitations.
The intricate photophysics of TADF emitters poses severe challenges for its computational description while also providing an ideal test bed for evaluating the newest computational tools. The importance of CT states already makes it clear that the application of time-dependent density functional theory (TDDFT) is challenging and expected to affect energies $\frac{10}{10}$ as well as state characters. 11 Suggestions for addressing this problem range from empirical corrections ${ }^{12}$ to optimal tuning of range-separated func-

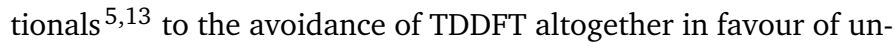
restricted ground-state DFT.6 Furthermore, state-specific excitedstate solvation effects have to be described at a high level considering that the common linear-response polarizable continuum model (LR-PCM) is inadequate for CT states with large electronhole separation ${ }^{14 \mid 15}$ and does not even provide any correction beyond zeroth order to triplet states due to their vanishing transition density. SOC also plays a role, having an intricate dependence on the state character where small amounts of mixed character lead to appreciable coupling. ${ }^{6}$ Finally, the importance of vibronic coupling between the locally excited (LE) and CT triplet states 1617 has been emphasised providing new dynamical routes to rISC.

A common theme in the above discussions is the importance of excited states of different character, in particular LE and CT states. Accurately discussing these states and the mixing between them can become a challenge on its own and a simple discussion of frontier orbitals is often inadequate. Thus, aside from the necessity of choosing an accurate electronic structure method, it becomes exceedingly important to choose a meaningful and reproducible method for analysing the computations. Indeed, a range of tools for categorising excited states are available. 
Categorisation of excited states occurs prominently via different measures of charge transfer,, 18 -20 which are a natural choice for push-pull systems as studied here. However, special care has to be taken for symmetric systems, including the molecules studied here, noting that the dipole moment and related charge displacement metrics are not reliable descriptors for the charge transfer character in symmetric systems. $\frac{192122]}{}$ A powerful option to overcome this problem amounts to base the analysis on a correlated electron-hole pair within an effective exciton picture; 23 and this was shown to be particularly suitable even for challenging cases with high symmetry. $24 \cdot 26$ Aside from categorising excited states, there has been an increasing push toward a rationalisation of the underlying energetics and, in particular, the singlet-triplet gap. The applied approaches range from a formal discussion of the underlying molecular orbital integrals ${ }^{115}$ to a direct extraction of computational data from actual electronic structure computations and applying it for energy decomposition and visualisation. 727228

It is the purpose of this work to exemplify the above discussion on four different TADF candidates highlighting their intricate photophysics as well as methodological challenges in the computational description. The four molecules studied are shown in Fig. 1, all possessing a D- $\pi$-A- $\pi$-D structure where a central accepting moiety (A) is symmetrically connected to two donors (D) whose separation is controlled by 1,4 -phenylene $\pi$-bridges. All molecules employ a carbazole $(\mathbf{C z})$ donor group and we use four different core groups, benzo[1,2-b:4,5-b']dithiophene-4,8-dione (BDT), anthraquinone (AQ), benzo[1,2-b:4,5-b']dithiophene-S,Sdioxide-4,8-dione (BDT-SO ${ }_{2}$ ), and benzo[1,2-b:4,5-b']difuran4,8-dione (BDF). Two of these molecules Cz-BDT ${ }^{29}$ and Cz-AQ ${ }^{4}$ have been previously synthesised and characterised. We will study these in detail moving on to predicting the properties of Cz-BDT-SO ${ }_{2}$ and Cz-BDF. Within this work, we contrast these four related molecules highlighting the profound changes in their observed photophysical properties following seemingly inconspicuous changes in the acceptor unit.

The employed acceptor unit BDT is an electron deficient ringfused heterocycle and a synthetic intermediate towards the synthesis of the fully aromatic and strongly-electron donating benzodithiophene moiety which is widely exploited in high performance donor polymers for organic solar cells. ${ }^{[30+36}$ While benzodithiophene has seen great utilisation as a donor, BDT itself has received much less attention as an acceptor unit despite its deep LUMO energy which is ca. $0.39 \mathrm{~V}$ lower than that of the carbocyclic homologue anthraquinone. 37138

Cz-BDT was designed to complement one of the best reported red TADF luminogens at the time, $\mathbf{C z}-\mathbf{A Q}, 4 \sqrt{4}$ and induce a redshift in emission due to the lower LUMO of BDT. This did lead to a $>100 \mathrm{~nm}$ redshift in emission wavelength compared to $\mathrm{Cz}$ $\mathbf{A Q}$, and TADF was evident from the characteristic double decay in the transient absorbance spectra, however the photoluminescence quantum yield (PLQY) was greatly reduced in both solution and dilute thin film measurements from $\approx 0.60$ for $\mathrm{Cz}-\mathrm{AQ}$ in solution to $<0.10$ for Cz-BDT. We note that in the meantime alternative strategies in the design of red TADF emitters have led to high PLQY extending into the near infrared range. 39,46

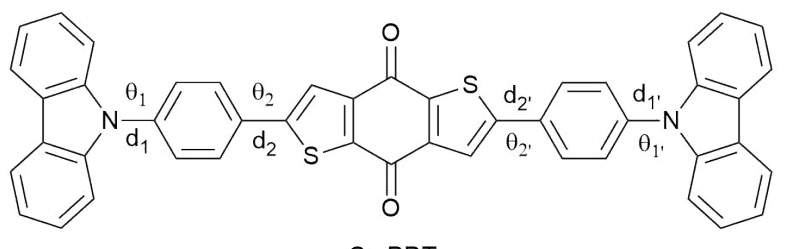

Cz-BDT
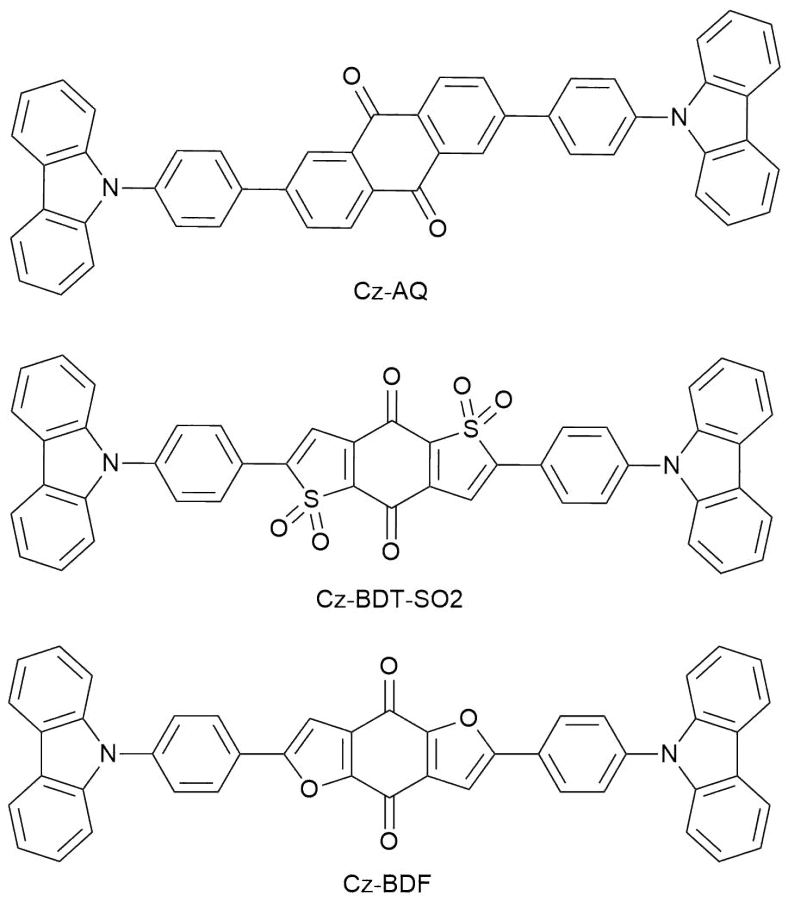

Fig. 1 The molecular structures of the four $D-\pi-A-\pi-D$ molecules studied within this work. The dihedral angles and bond distances discussed throughout are indicated by $\theta_{n}$ and $d_{n}$, and are defined in the same way for all molecules in this work.

Nonetheless, identifying the root cause of the vast drop in PLQY between Cz-AQ and Cz-BDT presents a useful challenge to inform future molecular design and to provide deep insight into the photophysics of TADF emitters along with methodological challenges in its computational description.

Here, we also study $\mathbf{C z}$-BDT-SO ${ }_{2}$ where dearomatisation of the fused heterocycles will serve to further reduce the LUMO (and to a lesser extent the HOMO) through inductive effects and remove the influence of the $\mathrm{S}$ lone pairs on the electronic properties of the molecule. Oxidation of $\mathrm{S}$ to $\mathrm{SO}_{2}$ serves to stabilise the LUMO and red-shift emission while it is also expected to enhance PLQYs and modulate singlet and triplet energies, $\Delta E_{S T}$, spin-orbit coupling effects, and solvatochromism. ${ }^{47-150}$ Finally, Cz-BDF is studied in order to identify the impact of light vs heavy heteroatoms on molecular geometries, excited state energies, and characters.

Within this work, we first explain the methods used discussing the general strategy employed for analysing excited-state wavefunctions and proceeding to specifics of the computational details. The results are presented in some detail starting with general structural parameters and frontier orbitals, proceeding to a discussion of the vertical excitations in terms of the state characters present and the computational description, finishing with 
an exploration of excited-state minima in solution. Before concluding, we proceed by a compact discussion summarising the photophysics, highlighting general differences between singlet and triplet state wavefunctions, and reviewing the most critical methodological aspects.

\section{Methods}

\subsection{Wavefunction analysis}

Analysis of excited state wavefunctions is performed via TheoDORE, ${ }^{51}$ which provides automatic, fragment-based assignment of state character. Specifically, the analysis uses the oneelectron transition density matrix (1TDM), which is formally defined as:

$$
\gamma_{0 I}\left(r_{h}, r_{e}\right)=n \int \ldots \int \Psi_{0}\left(r_{h}, r_{2}, \ldots, r_{n}\right) \Psi_{I}\left(r_{e}, r_{2}, \ldots, r_{n}\right) d r_{2} \ldots d r_{n}
$$

where $\Psi_{0}$ and $\Psi_{I}$ are the ground and excited state wavefunctions and $r_{h}$ and $r_{e}$ represent the coordinates of the excitation hole and the excited electron electron respectively. ${ }^{52}$ The excited state described by the 1TDM can be decomposed into local and CT contributions by computing the charge transfer numbers defined as the integral: $\underline{52[53}$

$$
\Omega_{A B}=\int_{A} \int_{B}\left|\gamma_{0 I}\left(r_{h}, r_{e}\right)\right|^{2} d r_{e} d r_{h}
$$

where the hole and electron are restricted to fragment $A$ and $B$ respectively. Practically, Eq. (2) is evaluated using a Mullikenstyle population analysis, $\frac{52}{2}$ here.

Excited states can also be analysed via the natural transition orbitals (NTOs), 54 computed through a singular value decomposition where the 1TDM is expressed as $\frac{52 \mid 53}{53}$

$$
\gamma_{0 I}\left(r_{h}, r_{e}\right)=\sum_{t} \sqrt{\lambda_{t}} \psi_{t}^{h}\left(r_{h}\right) \psi_{t}^{e}\left(r_{e}\right)
$$

Eq. (3) describes a sum over orbital pairs where $\psi_{t}^{h}$ and $\psi_{t}^{e}$ are the NTOs representing the hole and electron, and $\lambda_{t}$ is the amplitude of the transition. In the specific case that a state can be described by a single transition between two NTOs, in other words, the state is not multiconfigurational, the 1TDM can be factorised into a single pair of NTOs:

$$
\gamma_{0 I}\left(r_{h}, r_{e}\right)=\psi^{h}\left(r_{h}\right) \psi^{e}\left(r_{e}\right)
$$

and the charge transfer numbers (Eq. 2) are:

$$
\begin{gathered}
\Omega_{A B}=\int_{A} \int_{B}\left|\psi^{h}\left(r_{h}\right) \psi^{e}\left(r_{e}\right)\right|^{2} d r_{e} d r_{h} \\
=\int_{A}\left|\psi^{h}\left(r_{h}\right)\right|^{2} \times \int_{B}\left|\psi^{e}\left(r_{e}\right)\right|^{2} d r_{e}=q_{A}^{h} q_{B}^{e}
\end{gathered}
$$

Thus, for simple transitions involving only one orbital pair, the charge transfer numbers are completely determined by the NTOs and are a product of hole charges on fragment $\mathrm{A}, q_{A}^{h}$, and electron charges on fragment $\mathrm{B}, q_{B}^{e}$. In more general cases, the CT numbers also encode interference effects between the different excited configurations (cf. Ref. 22).

The CT numbers constitute a versatile tool and have been ap- plied successfully for, e.g., interacting chromophores, $\frac{55}{5}$ pushpull systems 26 and transition metal complexes. 56 57 However, a downside of this analysis is that it depends on the a priori definition of a fragmentation scheme. To overcome this problem and obtain a more fundamental measure of charge transfer, we compute an exciton size defined as the root-mean-square separation of electron and hole

$$
d_{\text {exc }}=\sqrt{\frac{\left\langle\gamma_{0 I}\left|\left(r_{h}-r_{e}\right)^{2}\right| \gamma_{0 I}\right\rangle}{\left\langle\gamma_{0 I} \mid \gamma_{0 I}\right\rangle}}
$$

where the bra/ket notation refers to integration with respect to $r_{h}$ and $r_{e} \cdot 23$ The exciton size, defined in this way, is a transferable measure that provides insight into charge transfer between isolated molecules, 23 covalently bonded donor-acceptor systems, 58 as well as large conjugated $\pi$-systems. $\stackrel{24}{ }$ Purely local excitations generally have a $d_{\text {exc }}$ of $4 \AA$ or less, where anything above this value indicates at least partial CT character. $7 / 24$

In addition we will utilise the absolute mean electron-hole separation

$$
d_{h e}=\left|\frac{\left\langle\gamma_{0 I}\left|\left(r_{h}-r_{e}\right)\right| \gamma_{0 I}\right\rangle}{\left\langle\gamma_{0 I} \mid \gamma_{0 I}\right\rangle}\right| .
$$

The $d_{h e}$ value, which is closely related to the dipole moment, measures the distance between the centroids of the hole and electron distributions. In the present context, $d_{h e}$ vanishes if both $\mathrm{D}$ units are equally involved in the excitation and only becomes significantly different from zero if there is a localisation of the excitation on one of them. It is, therefore, an ideal tool to monitor symmetry breaking in the excited state.

\subsection{Computational Details}

Computations are performed on Cz-BDT, Cz-AQ, Cz-BDT-SO and Cz-BDF as shown in Fig. 1. The tertiary butyl groups used to improve solubility in the experimental studies have been excluded.429 Computations in this study are divided into three parts: (i) an initial optimisation of the ground state geometry, (ii) vertical excitations with different functionals, and (iii) excitedstate geometry optimisations in solution.

For step (i) the molecular geometries of Cz-BDT and Cz-AQ were optimised at the $\omega \mathrm{B} 97 \mathrm{X}-\mathrm{V} / \mathrm{def} 2-\mathrm{SVP}$ level of theory 59.60 and confirmed as being minimum energy structures by a vibrational analysis at the same level. For step (ii), to determine an appropriate computational method to describe the excited state character of the molecules, the first 10 vertical excitations are first computed at the ri-ADC(2) level, 61 using the def2-TZVP basis set. $\frac{60[62}{6}$ The first 10 excited states are, then, recomputed using five different density functionals with TD-DFT in the TammDancoff approximation (TDA), 63 considering that the TDA is expected to reduce problems associated with triplet instabilities. 64 The density functionals evaluated are PBE, 65 PBE0, $66 \omega \mathrm{PBEh}, 67$ CAM-B3LYP, 68 70 and $\omega B$ 97X-V,, 59 with the def2-SV(P) ${ }^{60}$ basis set. In the case of $\omega \mathrm{PBEh}$, we used a range separation parameter of $\omega=0.1$ a.u. and a global amount of Hartree-Fock exchange of $20 \%$ following previous experience on related donor/acceptor systems. 47

The $\omega$ PBEh/def2-SV(P) level of theory is selected for step (iii) 
based on results from density functional benchmarking to the experimental absorption maximum and the ri-ADC(2) state characters. The ground state $\left(S_{0}\right)$ structures of all four molecules were optimised using spin-restricted Kohn-Sham DFT (RKS) along with the $\omega$ PBEh functional in toluene solution $(\varepsilon=2.3741)$ using a conductor-like polarisable continuum model (PCM). ${ }^{71 / 72}$ Excited singlet states $\left(S_{1}\right)$ were optimised using time-dependent density functional theory (TDDFT) along with the $\omega$ PBEh functional using a toluene solvent model $\left(\varepsilon=2.3741, \varepsilon_{\infty}=2.2403\right)$ using a linear response (LR-PCM) $73 \mid 74$ approach for excited-state solvation. Excited-triplet states $\left(T_{1}\right)$ were optimised at the TDDFT/LRPCM level of theory along with additional ground-state spinunrestricted Kohn-Sham DFT computations (UKS/PCM). No empirical dispersion correction is used as there is no clear way of choosing appropriate parameters for a manually adjusted functional and since preliminary observations showed that dispersion only has a minor effect on these linear molecules.

Based on the $S_{0}$ optimised geometries, we compute the TDDFT vertical excitation energies of the first 7 excited states using two different solvation methods, LR-PCM and a perturbative statespecific solvation model (pt-SS). ${ }^{15 / 75}$ At the excited-state geometries we perform TDDFT computations using the LR-PCM and SSPCM $14 / 75 / 76$ models. Specifically, the following workflow is used: All three PCM approaches start with a RKS/PCM ground-state calculation. LR-PCM, which is the default approach, proceeds with a TDDFT computation including a correction for non-equilibrium solvation added directly to the TDDFT response matrix. The ptSS approach, on the other hand, starts with a zeroth order TDDFT response matrix - not including any corrections for solvation. It proceeds by computing a state-specific correction term based on the zeroth order response vector and the relaxed ground state density. Oscillator strengths (f) were computed as $f=2 / 3 \times \Delta E \times \mu^{2}$ by combining the original transition dipole moments $\mu$ with the perturbatively corrected excitation energies $\Delta E$. Finally, SS-PCM is technically carried out via two subsequent TDDFT jobs: in the first step the solvent field is equilibrated to the state of interest and in the second step the DFT orbital optimisation as well as TDDFT are carried out in the equilibrated solvent field.

All (TD)DFT computations were performed with Q-Chem 5.2. ${ }^{77}$ ri-ADC(2) computations used Turbomole 7.2. ${ }^{78}$ Wavefunction analysis, following the ideas outlined in Section 2.1, were carried out in TheoDORE 2.3. $51 / 53$

The underlying research data (molecular geometries, input/output files of Q-Chem and Turbomole) is provided via a separate repository. 79

\section{Results}

\subsection{Molecular structures and frontier orbitals}

The D- $\pi$-A- $\pi$-D molecules studied here are comprised of carbazole (Cz) donor units, phenylene ( $\mathbf{P h}$ ) ring $\pi$ bridges, and the acceptor core (BDT or AQ). These molecules have a significant degree of conformational flexibility because rotation is possible around the carbazole-phenyl bonds and the phenyl-core bonds. This gives rise to four independent dihedral angles, denoted as $\theta_{1} / \theta_{1}^{\prime}$ and $\theta_{2} / \theta_{2}^{\prime}$ (see Fig. 1). To restrict the associated number of conform- ers, we will consider only molecules of $C_{2}$ symmetry $\left(\theta_{1}=\theta_{1}^{\prime}\right.$, $\left.\theta_{2}=\theta_{2}^{\prime}\right)$ and $C_{i}$ symmetry $\left(\theta_{1}=-\theta_{1}^{\prime}, \theta_{2}=-\theta_{2}^{\prime}\right)$. Furthermore, we find that the energy difference between the $\mathrm{C}_{i}$ and $\mathrm{C}_{2}$ conformers is negligible (see Table S1, ESI) indicating that both, along with other conformers, should be present at room temperature. To obtain consistent results, we select $\mathrm{C}_{2}$ conformers for all subsequent calculations, unless specified explicitly.
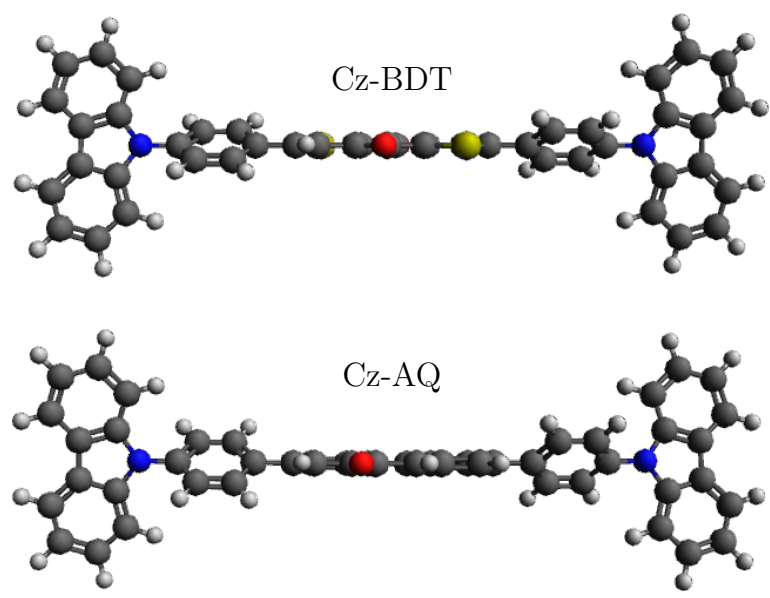

Fig. 2 The 3D structures of $C z-B D T$ and $C z-A Q$ optimised at the $\omega \mathrm{B} 97 \mathrm{X}-\mathrm{V} /$ def2-SVP level of theory in $\mathrm{C}_{2}$ symmetry.

Optimised ground-state geometries, considering the $C_{2}$ case, are presented in Fig. 2 and the geometric data for $C_{2}$ and $C_{i}$ are shown in Table 1 . Generally speaking, we find significant twisting for both torsion angles considered. Starting with the discussion of the $C_{2}$ geometries, the $\mathbf{C z} / \mathbf{P h}$ twisting angles $\left(\theta_{1} / \theta_{1}^{\prime}\right)$ are consistently $\approx 57^{\circ}$ for both molecules. By comparison, the twisting around the $\mathbf{P h}$ /core junction $\left(\theta_{2} / \theta_{2}^{\prime}\right)$ is notably reduced owing to reduced steric hindrance. Twisting in Cz-AQ is larger than in $\mathbf{C z}-$ BDT ( $39^{\circ}$ vs $26^{\circ}$ ) which can be understood by considering that the AQ group has two hydrogen atoms in the vicinity of the $\mathbf{P h}$ group whereas BDT only has one. Cz-BDT is, thus, expected to allow for enhanced conjugation between the Ph and BDT groups and we will explore the consequences of this on the observed photophysics in more detail below. Proceeding to the bond distances, we find that the $\mathbf{C z}-\mathbf{P h}$ distances $\left(d_{1} / d_{1}^{\prime}\right)$ are very similar for both molecules $\left(\approx 1.417 \AA\right.$ ). For the $d_{2} / d_{2}^{\prime}$ values however, the bond distance is shorter in Cz-BDT (1.479 $\AA$ ) than Cz-AQ (1.491 $\AA$ ) following the same trends as expected for the bond angles, i.e. conjugation is stronger for Cz-BDT. Table 1 also presents results for the $C_{i}$ geometry as well as data from previous work on the two molecules ${ }^{4 / 29}$ using the B3LYP functional. The observed trends between the three data sets are consistent indicating that these geometric parameters are fairly robust with respect to both the precise conformer studied and the functional chosen.

To discuss the electronic structure of this system, we start with the frontier molecular orbitals as is usually done for these types of molecules. The highest occupied molecular orbital (HOMO) and lowest unoccupied MO (LUMO) for Cz-BDT are shown in Fig 3 and for Cz-AQ in Fig S1 (ESI). The HOMO and LUMO look the same for both molecules; the HOMO is located on the carbazole 
Table 1 Twist angles and bond distances for BDT and AQ molecules obtained at the $\omega$ B97X-V/def2-SVP level of theory. Results from Refs 29 and 4 are shown for comparison.

\begin{tabular}{lccccc}
\hline & Molec. & $\theta_{1} / \theta_{1}^{\prime}$ & $d_{1} / d_{1}^{\prime}$ & $\theta_{2} / \theta_{2}^{\prime}$ & $d_{2} / d_{2}^{\prime}$ \\
\hline$C_{2}$ data & Cz-BDT & 56.5 & 1.417 & 26.0 & 1.479 \\
$C_{i}$ data & & 56.7 & 1.417 & 29.9 & 1.479 \\
Ref. 29 & & 50 & 1.415 & 24 & 1.470 \\
\hline$C_{2}$ data & Cz-AQ & 57.6 & 1.418 & 38.7 & 1.491 \\
$C_{i}$ data & & 57.2 & 1.418 & 38.1 & 1.491 \\
Ref. 4 & & 50 & 1.412 & 36 & 1.490 \\
\hline
\end{tabular}

donor units and the LUMO is located on the acceptor core and there are only negligible contributions on the bridging $\mathbf{P h}$ units. A transition from the HOMO to the LUMO would, therefore, produce an excited state with a large amount of charge transfer (CT) character, shifting electron density from the donor to the acceptor. However, HOMO/LUMO plots can only ever provide a very rough picture of the electronic structure and we will proceed to a detailed analysis of the excited states and all orbitals involved, below.

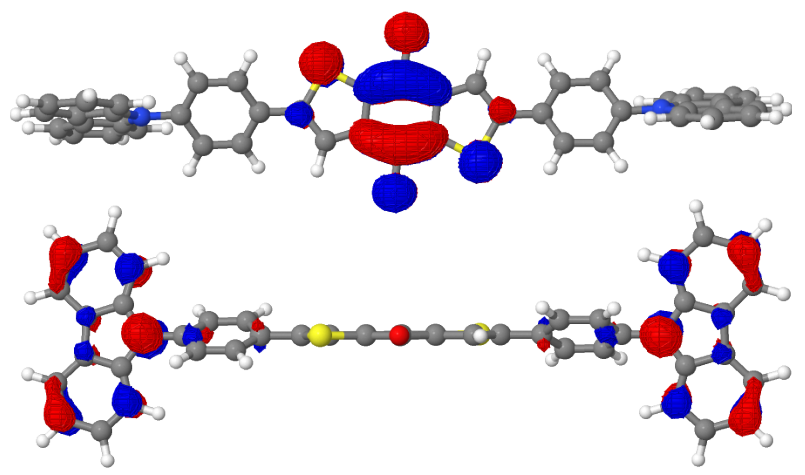

Fig. 3 The HOMO (bottom) and LUMO (top) for Cz-BDT calculated at the $\omega \mathrm{B} 97 \mathrm{X}-\mathrm{V} /$ def2-SVP level of theory.

\subsection{Vertical excitations}

Proceeding to the excited states of Cz-BDT and Cz-AQ, we first endeavour to find a computational method that describes their states accurately. To do so, we consider experimental results as well as reference calculations at the $a b$ initio ri-ADC(2)/TZVP level of theory. Experimental results are presented in Table $2 . \mathbf{~ C z}-$ BDT is found to have an absorption maximum at $2.65 \mathrm{eV}$ and a strongly red-shifted emission at $1.87 \mathrm{eV}$. In the case of Cz-AQ both values are about $0.3 \mathrm{eV}$ higher. Table 2 also highlights the considerable difference of the fluorescence quantum yield $\Phi$ measured in solution between the two molecules, showing a more than sixfold drop when going from Cz-AQ to Cz-BDT. Whereas, the shift in absorption and emission maxima can be explained by the lowering of the LUMO, it is harder to gain understanding of the drop in quantum yield.

To obtain computational reference data, we compute the first ten excited states of Cz-BDT and Cz-AQ at the ri-ADC(2) level in
Table 2 Experimentally measured photophysical data for $\mathrm{Cz}-\mathrm{BDT}$ and Cz-Aq: Energies for the first absorption and emission maxima $\left(E_{a b s, \max }\right.$, $\left.E_{\text {em, } \max }\right)$ and photoluminescence quantum yield $(\Phi)$.

\begin{tabular}{lccc}
\hline & $E_{a b s, \max }(\mathrm{eV})$ & $E_{\text {em, } \max }(\mathrm{eV})$ & $\Phi$ \\
\hline $\mathbf{C z}-\mathrm{BDT}^{\mathrm{a}}$ & 2.65 & 1.87 & 0.095 \\
$\mathbf{C z}^{\mathrm{A}} \mathbf{A Q}^{\mathrm{b}}$ & 2.91 & 2.22 & 0.60 \\
\hline
\end{tabular}

${ }^{a}$ Ref. 29, measured in toluene

${ }^{\mathrm{b}}$ Ref. 4, measured in toluene

(a)

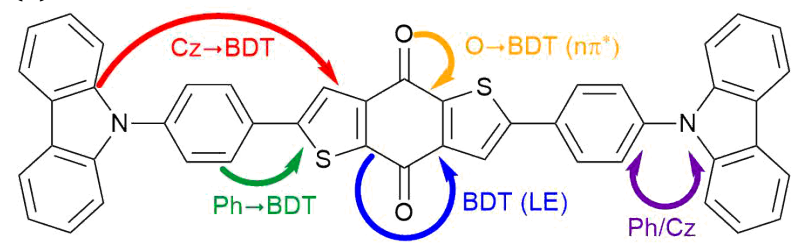

(b)

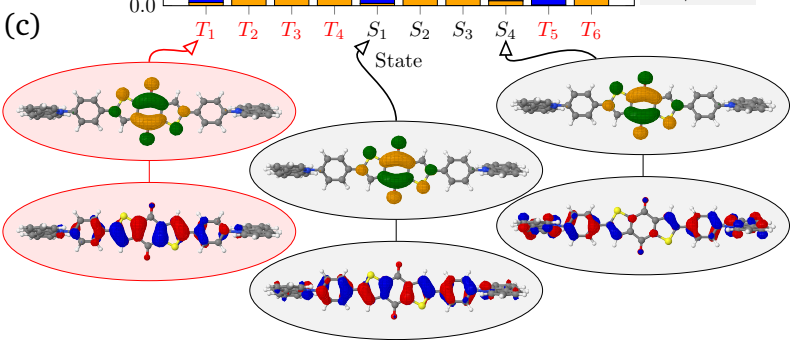

Fig. 4 Analysis of excited states in Cz-BDT at the ri-ADC(2)/TZVP level of theory: (a) Schematic of the fragment based decomposition, (b) excited state energies for singlets in black and triplets in red with oscillator strengths as shading (top) and characters (bottom) of the first ten excited states of Cz-BDT, and (c) natural transition orbitals (NTOs) for selected states.

gas phase at the ground state $C_{2}$ geometries. To show the results of these computations, we use a graphical representation, developed in Refs 25 51, that provides compact information about excited energies and characters based on the CT numbers [Eq. (2)] and the natural transition orbitals [Eq. (3)]. For the CT number analysis, the molecule is first divided into different fragments. In the case of Cz-BDT, these are (i) the $\mathbf{C z}$ donors, (ii) the phenylene (Ph) bridges, (iii) the BDT core, and (iv) the oxygen groups on the core. Using these fragments, we can now decompose the excited states into different contributions as shown in Fig. 4 (a). We will consider the following five contributions:

- $\mathbf{C z} \rightarrow$ BDT charge transfer (red),

- $\mathbf{P h} \rightarrow$ BDT charge transfer (green),

- local BDT excitations (blue), 
(a)

- excitations from the oxygen atoms to BDT indicating $n \pi *$ character (orange), and

- excitations within the $\mathbf{C z}$ and Ph units (purple).

These are shown schematically in Fig. 4(a) and a detailed definition of the different contributions is given in Figure S2 (ESI). Any excited state is a combination of these elementary contributions in varying weights and we will use bar graphs to represent the decomposition of the individual states.

The excitation energies for Cz-BDT are presented in the upper panel in Fig. 4(b) showing that this molecule possesses four lowenergy triplet states (red) before the first singlet state (black). Viewing the lower panel of Fig. 4 (b), we find that $T_{1}$ is dominated by local $\pi \pi^{*}$ character on the BDT core, represented in blue, with smaller $\mathbf{P h} \rightarrow$ BDT CT contributions (green). The corresponding NTOs are shown in panel (c) highlighting that the hole NTO (shown in blue/red) is a $\pi$-orbital on BDT with some contributions on the Ph bridge whereas the electron NTO (shown in green/orange) is a $\pi^{*}$-orbital located right at the centre of BDT resembling the LUMO shown in Fig. 3 Proceeding to the next three triplet states, we find that these are a mixture of local $\pi \pi^{*}$ character on BDT (blue) and $n \pi^{*}$ character (orange) with increasing $\mathrm{n} \pi^{*}$ character from $T_{2}$ to $T_{4}$. The first singlet state $\left(S_{1}\right)$ lies just above $3.0 \mathrm{eV}$ and is similar in character to $T_{1}$ albeit with more charge transfer character (red and green). This is also reflected by the dominant hole NTO, shown at the bottom in panel (c), which has extended contributions on the $\mathbf{P h}$ bridges. The vertical gap between $T_{1}$ and $S_{1}$ is calculated to be $0.42 \mathrm{eV}$. The $S_{2}$ and $S_{3}$ states are locally excited states dominated by $\mathrm{n} \pi^{*}$ character. The $S_{4}$ state lying at $3.28 \mathrm{eV}$ is a CT state which contains significant $\mathbf{C z} \rightarrow$ BDT character (red). For the $T_{5}$ and $T_{6}$ states, we find a mixture of state characters with roughly $40 \%$ of the excitation character attributed to local excitations on $\mathbf{P h}$ and $\mathbf{C z}$.

Reviewing the states in the canonical orbital picture, we find that all states go predominantly into the LUMO, with only the $T_{5}$ and $T_{6}$ states also containing significant contributions into higher virtual orbitals (LUMO+ 1 and LUMO+3). Conversely, the HOMO only plays a minor role for the low energy states and of all the states considered here, only $S_{1}$ and $S_{4}$ and none of the triplet states have notable HOMO-LUMO character (62\% for $S_{1}, 85 \%$ for $S_{4}$ ). This highlights that a simple visualisation of the HOMO and LUMO can by no means explain the photophysics of complicated TADF systems as studied here.

The oscillator strengths are represented via the colour shading in the upper panel of 4 (b). At the highly symmetric geometry shown, the first bright state is $S_{4}$ at $3.28 \mathrm{eV}$, with an oscillator strength of 0.45 . The lowest $\pi \pi^{*}$ state, $S_{1}$ at $3.06 \mathrm{eV}$, possesses vanishing oscillator strength within the computation. This can be understood by the fact that the analogous state is symmetry forbidden under $C_{i}$ symmetry and assuming that similar orbital interactions are also present at the $C_{2}$ geometry. However, once the symmetry is broken, this state is expected to borrow intensity from the bright state suggesting that this state contributes to the lowest energy band in the absorption spectrum, which is found experimentally at $2.65 \mathrm{eV}$.

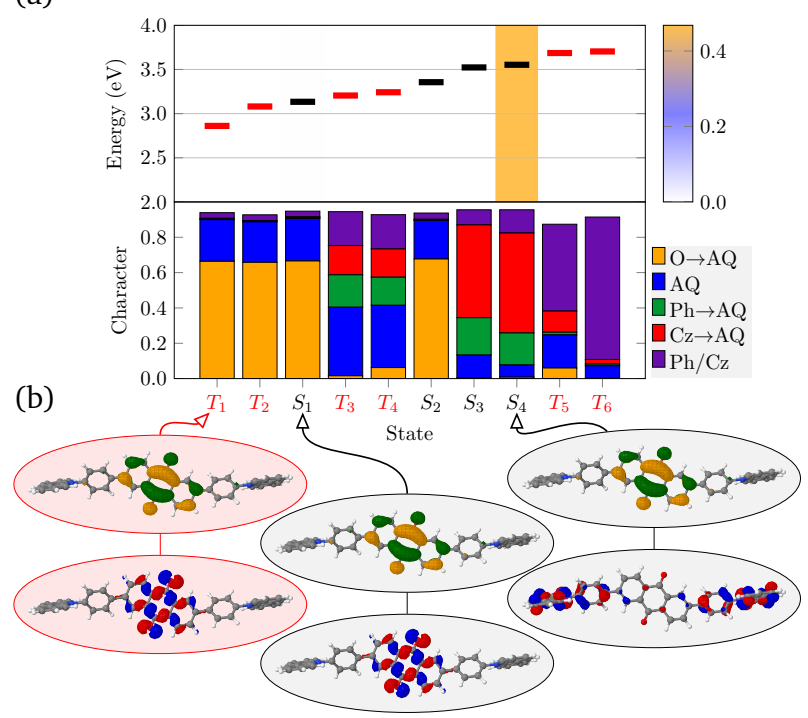

Fig. 5 Analysis of excited states in Cz-AQ at the ri-ADC(2)/TZVP level of theory: (a) excited state energies for singlets in black and triplets in red with oscillator strengths as shading (top) and characters (bottom) of the first ten excited states of $\mathrm{Cz}-\mathrm{BDT}$, and (b) natural transition orbitals (NTOs)

Next, we move to the second molecule studied here, Cz-AQ. As opposed to Cz-BDT where the lowest state was of local $\pi \pi^{*}$ character, we find in the case of Cz-AQ that the first three states $\left(S_{1}, T_{1}, T_{2}\right)$ are all of $\mathrm{n} \pi^{*}$ character (orange). The calculated vertical gap between $S_{1}$ and $T_{1}$ is $0.32 \mathrm{eV}$ which is $0.1 \mathrm{eV}$ smaller than found in Cz-BDT. The $T_{3}$ and $T_{4}$ states are mixed in character, with mostly local contributions on $\mathbf{A Q}$ and smaller amounts of charge transfer from $\mathbf{P h}$ and $\mathbf{C z}$ to the core. The $S_{2}$ state is an $n \pi *$ state. $S_{3}$ and $S_{4}$ are both described as charge transfer states with significant $\mathbf{C z} \rightarrow$ BDT character. Only $S_{4}$ has an appreciable oscillator strength, which can, again, be understood in terms of symmetry properties. The $S_{4}$ excitation energy is $3.55 \mathrm{eV}$ which is $0.64 \mathrm{eV}$ higher than the experimentally determined absorption maximum, thus ri-ADC(2) overestimates the energy of the bright state by about $0.5 \mathrm{eV}$ for both Cz-BDT and Cz-AQ. Conversely, ri$\mathrm{ADC}(2)$ is expected to underestimate the energies of $n \pi *$ states by a few tenths of an $\mathrm{eV} \cdot[\sqrt[60]{ }$ Nonetheless, we believe that ri-ADC(2) provides a good reference for the expected state characters involved. In particular, these results indicate that all types of states, i.e., local $\pi \pi^{*} / \mathrm{n} \pi^{*}$ states and CT states, play a role.

Finally, viewing the difference between Figures 4 and 5 , we find that the $S_{1}$ and $T_{1}$ states of Cz-BDT have significant locally excited $\pi \pi^{*}$ contributions on BDT whereas $S_{1}$ and $T_{1}$ are dominated by $\mathrm{n} \pi^{*}$ character for Cz-AQ. Proceeding to the higher excited states, we find enhanced CT (red) for Cz-AQ. Thus, we can already anticipate that the photophysics of $\mathbf{C z}-\mathbf{A Q}$ will be dominated by its CT states whereas locally excited $\pi \pi^{*}$ contributions are more important for Cz-BDT.

Having described the states at the ab initio ri-ADC(2) level, it is of interest whether an approximate density functional can be used with the aim of both saving computational time, and to find a method that matches closer with experimental absorption 


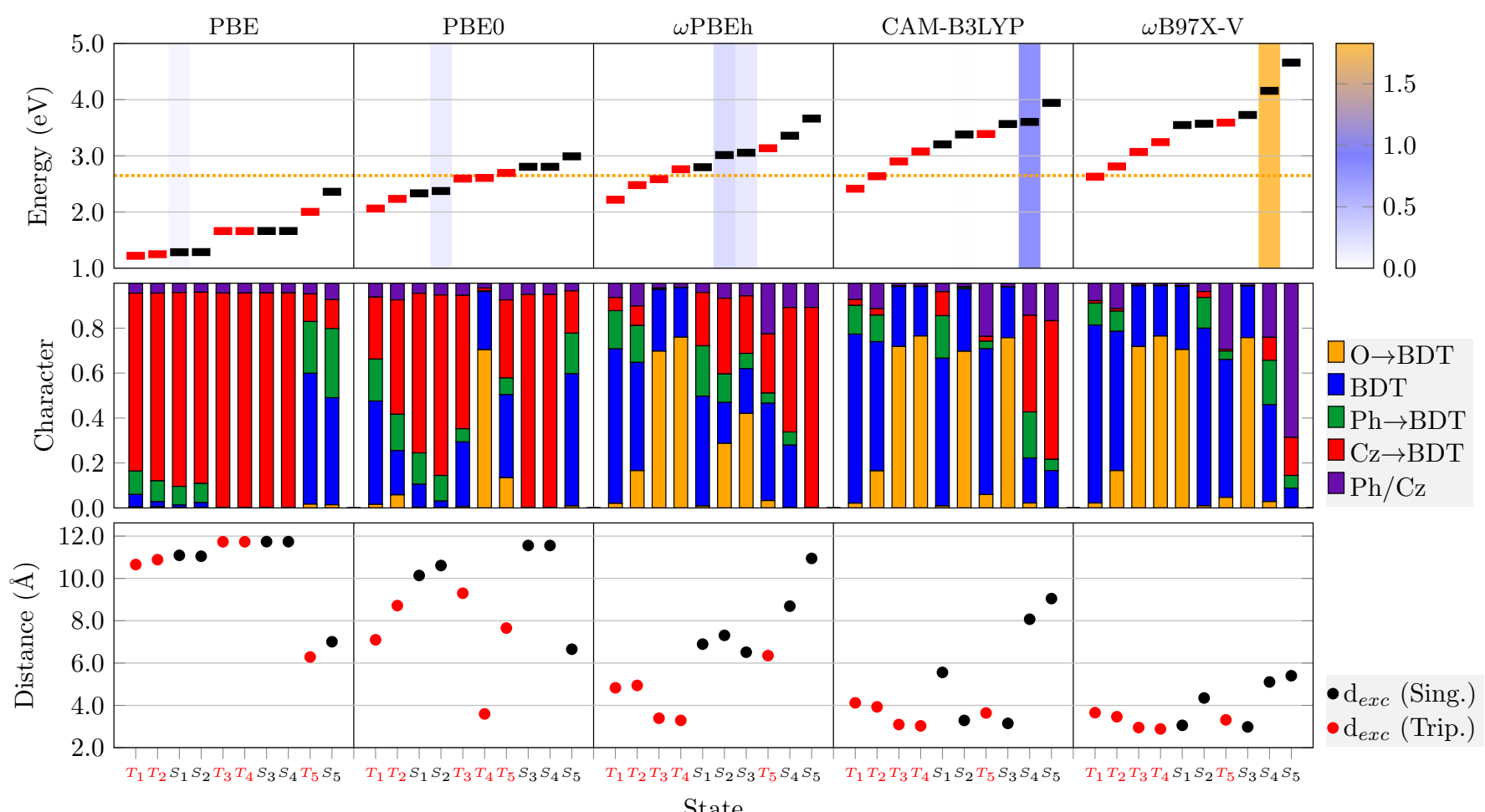

Fig. 6 Analysis of the first five singlet and triplet states of $\mathrm{Cz}-\mathrm{BDT}$ in $C_{2}$ symmetry computed using five different functionals (displayed in the top row) in vacuum. Top: excited-state energies (singlets black, triplets red) along with the experimental absorption maximum as dotted orange line and oscillator strengths shown as shading; middle: excited-state characters; bottom: exciton size $d_{e x c}$.

wavelengths. For this purpose, the first five singlet and triplet states for Cz-BDT and Cz-AQ are calculated using TDDFT with the PBE, PBE0, $\omega$ PBEh, CAM-B3LYP and $\omega$ B97X-V functionals. Figures 6 and S3 (ESI) contain the results for Cz-BDT and CzAQ respectively, with the state characters assigned as explained in 4 (a). The experimental absorption maximum is shown as a dashed orange line. The exciton size $\left(d_{\text {exc }}\right)$ for the excited state [Eq. 6], an alternative measure for charge transfer, 23|24 is shown in the bottom panel. In Fig. 6 the functionals are arranged according to an effective increase in Hartree-Fock exchange from left to right: PBE (0\%), PBE0 (25\%), $\omega$ PBEh (20100\%, $\omega=0.1$ bohr $^{-1}$ ), CAM-B3LYP (19-65\%, $\mu=0.33$ bohr $^{-1}$ ) and $\omega$ B97X-V $\left(16-100 \%, \omega=0.3\right.$ bohr $\left.^{-1}\right)$ where the amount of Hartree-Fock exchange (HFX) and the range-separation parameter $(\omega / \mu)$ are given in parentheses. Overall, with increasing HFX we find that the state energies, vertical singlet-triplet gaps, and the oscillator strengths of bright states increase.

More strikingly, the middle panel in Fig. 6 reveals the dramatic difference in the state characters obtained with the different functionals. In the case of PBE on the left, the first 8 states are almost entirely of $\mathbf{C z} \rightarrow \mathbf{B D T}$ CT character (red). By contrast, any substantial CT is missing for $\omega \mathrm{B} 97 \mathrm{X}-\mathrm{V}$ on the right. Only the three functionals in the middle contain the mixture of local $\pi \pi^{*} / \mathrm{n} \pi^{*}$ states and CT states as found for ri-ADC(2). The same trend is also found for the exciton sizes in the lower panel. The first eight states for PBE show enhanced charge separation $\left(d_{\text {exc }}>10 \AA\right)$ whereas no state with an exciton size above $6 \AA$ is found for $\omega \mathrm{B} 97 \mathrm{X}-\mathrm{V}$. And, again, more diverse values are found for the functionals in between. The difference in state character is reflected by changes in oscillator strength, shown as shading in the top panel of Fig. 6, which is strongly increased for the functionals with more HFX. These differences highlight the charge transfer problem in TDDFT showing that varying amount of HFX does not only affect the energies $\frac{10 \mid 81}{10}$ but also excitedstate properties. ${ }^{24 \mid 82}$ It is noteworthy, here, that a larger amount of "exact" exchange does not necessarily produce better agreement with higher-level computational methods considering that a reduced amount of exchange corresponds to a physically meaningful screened Coulomb interaction, cf. Ref. 7 .

We proceed to detailed results for the individual functionals. For PBE we find two sets of four almost degenerate CT states (two singlets and two triplets). These can be understood in the sense that they are composed of independent $\mathbf{C z} \rightarrow \mathbf{B D T}$ transitions on the left and right hand side of the molecule, which are effectively decoupled and neither split via Coulomb nor exchange interactions. The exciton sizes are all above $10 \AA$, which is similar to the distance from the centre of the $\mathbf{C z}$ donor to the centre of the BDT acceptor highlighting that charge transfer between them dominates with no intermediate locally excited contributions playing a role. Considering the global hybrid PBE0, we still find significant amounts of CT character for the first four states but there is already much more structure when compare to PBE. In particular, it is found that the $T_{1}$ state has enhanced local character (blue) and an associated reduction in exciton size to $7.1 \AA$. Moving to $\omega \mathrm{PBEh}$, we find that the first four states are triplets of local $\pi \pi^{*}$ and $n \pi^{*}$ character. These are followed by three sin- 
glet states around $3 \mathrm{eV}$ with a mixture of local $\pi \pi^{*}$ and $\mathrm{n} \pi^{*}$ as well as CT character. We find two bright states at 3.01 and 3.06 $\mathrm{eV}$, which possess significant amounts of, both, $\mathrm{n} \pi^{*}$ and CT character. This should be understood in the sense that there is an accidental degeneracy between an $\mathrm{n} \pi^{*}$ and CT state, producing this mixing. Moving to the remaining two functionals, we find that the first state with appreciable CT character is above $3.5 \mathrm{eV}$ for CAM-B3LYP and no CT state is found at all for $\omega$ B97X-V within the energy window considered (up to $4.5 \mathrm{eV}$ ).

For Cz-AQ (Figure S3, ESI), we find broadly the same story: PBE and PBE0 overestimate the amount of CT character of the lowest lying states and underestimate the energy of the bright state, while $\omega \mathrm{B} 97 \mathrm{X}-\mathrm{V}$ underestimates the CT character and severely overestimates the energy of the bright state. The main differences between $\omega$ PBEh and CAM-B3LYP are the energy of the bright singlet CT state, with $\omega$ PBEh providing a better value relative to the experimental absorption maximum, and the ordering of the states with respect to the state character. With the $\omega$ PBEh functional, we find a similar mixture of local $\pi \pi^{*} / \mathrm{n} \pi^{*}$ and CT states as for ri-ADC(2). The only difference is that the relative energies of the $n \pi^{*}$ states are somewhat raised yielding a different ordering of the dark low-energy states. However, as discussed above, ri-ADC(2) is expected to slightly underestimate $n \pi^{*}$ state energies, thus supporting the description by $\omega$ PBEh.

For both Cz-BDT and Cz-AQ, the $\omega$ PBEh functional gives a vertical excitation energy for the lowest bright state at a value in close proximity to the experimental value (indicated as dotted orange line in Figures 6 and S1). Here, experience suggests that due to vibronic effects, the vertical excitation should be about $0.1 \mathrm{eV}$ above the experimental maximum, 83 therefore indicating excellent agreement of the computed values. Furthermore, $\omega$ PBEh succeeds in describing the state characters of the low-energy states involved when compared to the higher-level ri-ADC(2) method and is expected to capture the overall photophysics well. Therefore, we will proceed by using the $\omega$ PBEh functional in the further course of the study.

\subsection{Excited state minima and solvation}

Having described the vertical excitations and identifying $\omega$ PBEh as a method for providing the overall excited state characters effectively, we now turn to an analysis of the excited state minimum geometries in solution for Cz-BDT and Cz-AQ. First, the geometries of the $\mathrm{S}_{0}, \mathrm{~S}_{1}$, and $\mathrm{T}_{1}$ minima are optimised using $\omega$ PBEh/def2-SVP within toluene solvation, considering that toluene was used in the experimental studies considered. ${ }^{4 / 29}$ The $S_{1}$ is always optimised with TDDFT whereas, following Ref. 6 , we investigate two possibilities for optimising the $T_{1}$ minimum, an excited-state optimisation using TDDFT and a formal groundstate optimisation using UKS. The key structural parameters are outlined in Table 3, and the geometries are shown in Fig. 7 Generally speaking, we find that upon excitation the molecules planarise, i.e. dihedral angles become smaller, and that the interring distances become shorter. Cz-BDT retains the two-fold symmetry in all computations whereas we observe symmetry-breaking in the excited state for Cz-AQ. Starting with Cz-BDT, we find that variations in the $\mathbf{C z}-\mathbf{P h}$ torsion $\theta_{1} / \theta_{1}^{\prime}$ are relatively minor whereas the $\theta_{2} / \theta_{2}^{\prime}$ values become close to zero for $S_{1}$ and $T_{1}$. This means that the Ph-BDT-Ph system becomes almost planar after photoexcitation, see also Figure 7, effectively producing one extended $\pi$-system, and we will revisit this point below. In Cz-Aq on the other hand, we find clear evidence of symmetry-breaking for the excited state minima: the angles on one side of the molecule $\left(\theta_{1}^{\prime}\right.$ and $\theta_{2}^{\prime}$ ) remain at values close to the $S_{0}$ minimum, however $\theta_{1}$ and $\theta_{2}$ are reduced. The $T_{1}$ minimum for $\mathbf{C z}-\mathbf{A Q}$, optimised with either TDDFT or UKS, has one of the Ph bridges almost in plane with the core, whilst for the $S_{1}$ minimum this lies at an intermediate value between the $S_{0}$ and $T_{1}$ minima.

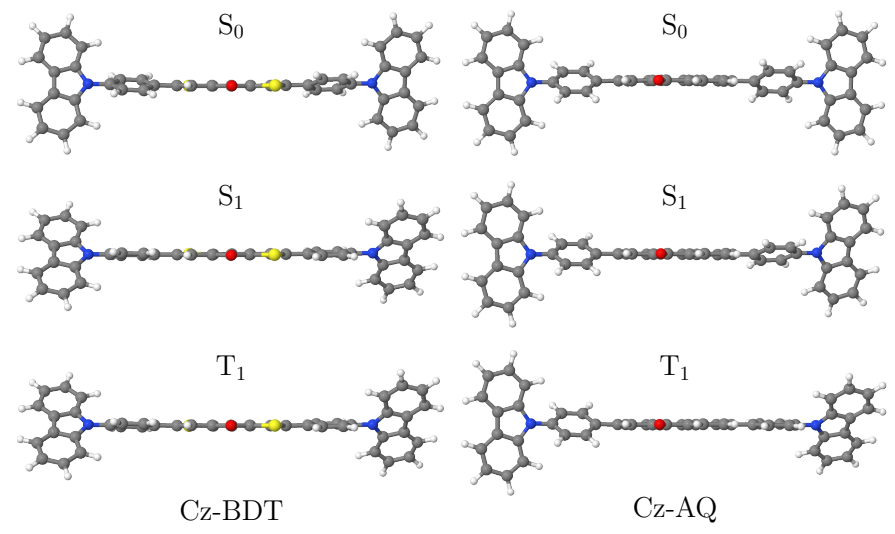

Fig. 7 The $\mathrm{S}_{0}, \mathrm{~S}_{1}$ and $\mathrm{T}_{1}$ minimum geometries of $\mathrm{Cz}-\mathrm{BDT}$ and $\mathrm{Cz}-\mathrm{AQ}$ optimised at the $\omega$ PBEh/def2-SVP level of theory.

We proceed by discussing the excited states at the individual geometries. For this purpose, we consider two different approaches, the standard LR-PCM method also used for the TDDFT optimisations, as well as a more involved state-specific approach, which is expected to provide an improved description of CT states. ${ }^{14 \mid 15}$ For the vertical absorption $\left(S_{0}\right)$, we use the perturbative (ptSS) approach whereas for emission $\left(S_{1} / T_{1}\right)$ we use the equilibrated SSPCM approach. Starting the discussion with Cz-BDT, we present data for the lowest four triplet and three singlet excited states in Figure 8. In comparison to the unsolvated model in Fig 6, we find similar state characters when using the LR-PCM model at the $S_{0}$ geometry for Cz-BDT. However, we find that the $\pi \pi^{*}$ states (blue, green, red) are somewhat shifted down in energy producing a different ordering of the states. This shift also removes the accidental degeneracy found between the bright $\pi \pi^{*}$ state and the $\mathrm{n} \pi^{*}$ state that was seen in the unsolvated calculation. Calculating the same excitations under the ptSS solvation scheme gives stabilisation to states with charge transfer character, and we now find the bright CT-dominated $S_{2}$ state at $2.71 \mathrm{eV}$ which is in nearperfect agreement with the experimental absorption maximum of $2.65 \mathrm{eV}$.

Next, we proceed to the optimised minimum of the $S_{1}$ state. The $S_{1}$ energy is found at an adiabatic energy of 2.37 and $2.36 \mathrm{eV}$ using LR-PCM and SS-PCM, respectively. The obtained emission energy is $2.19 \mathrm{eV}$. We find that the $S_{1}$ state at the $S_{1}$ minimum geometry has significant local BDT character (blue, $56 \%$ at SSPCM level) and a small exciton size of $6.20 \AA$, which is slightly 
Table 3 Geometric parameters for Cz-BDT and Cz-Aq obtained at the $\omega$ PBEh/def2-SVP level of theory with a toluene PCM solvent model. Only symmetry-unique values are given.

\begin{tabular}{ccccccc}
\hline Molecule & Geometry & Method & $\theta_{1} / \theta_{1}^{\prime}$ & $d_{1} / d_{1}^{\prime}$ & $\theta_{2} / \theta_{2}^{\prime}$ & $d_{2} / d_{2}^{\prime}$ \\
\hline Cz-BDT & & & & & & \\
& $S_{0} \min$ & RKS & -55.4 & 1.412 & -16.4 & 1.467 \\
& $S_{1} \min$ & TDDFT & -48.1 & 1.402 & 3.7 & 1.450 \\
& $T_{1}$ min & TDDFT & -49.0 & 1.404 & 4.0 & 1.447 \\
& $T_{1}$ min & UKS & -48.8 & 1.403 & 4.0 & 1.447 \\
Cz-AQ & & & & & & \\
& & & & & \\
& $S_{0}$ min & RKS & 57.6 & 1.418 & 38.7 & 1.491 \\
& $S_{1}$ min & TDDFT & $-43.6 /-57.7$ & $1.388 / 1.416$ & $-18.5 /-33.3$ & $1.460 / 1.483$ \\
& $T_{1}$ min & TDDFT & $-43.9 /-57.0$ & $1.390 / 1.415$ & $2.4 /-35.5$ & $1.433 / 1.483$ \\
& $T_{1}$ min & UKS & $-43.1 /-57.6$ & $1.388 / 1.415$ & $2.4 /-35.6$ & $1.433 / 1.483$ \\
\hline
\end{tabular}
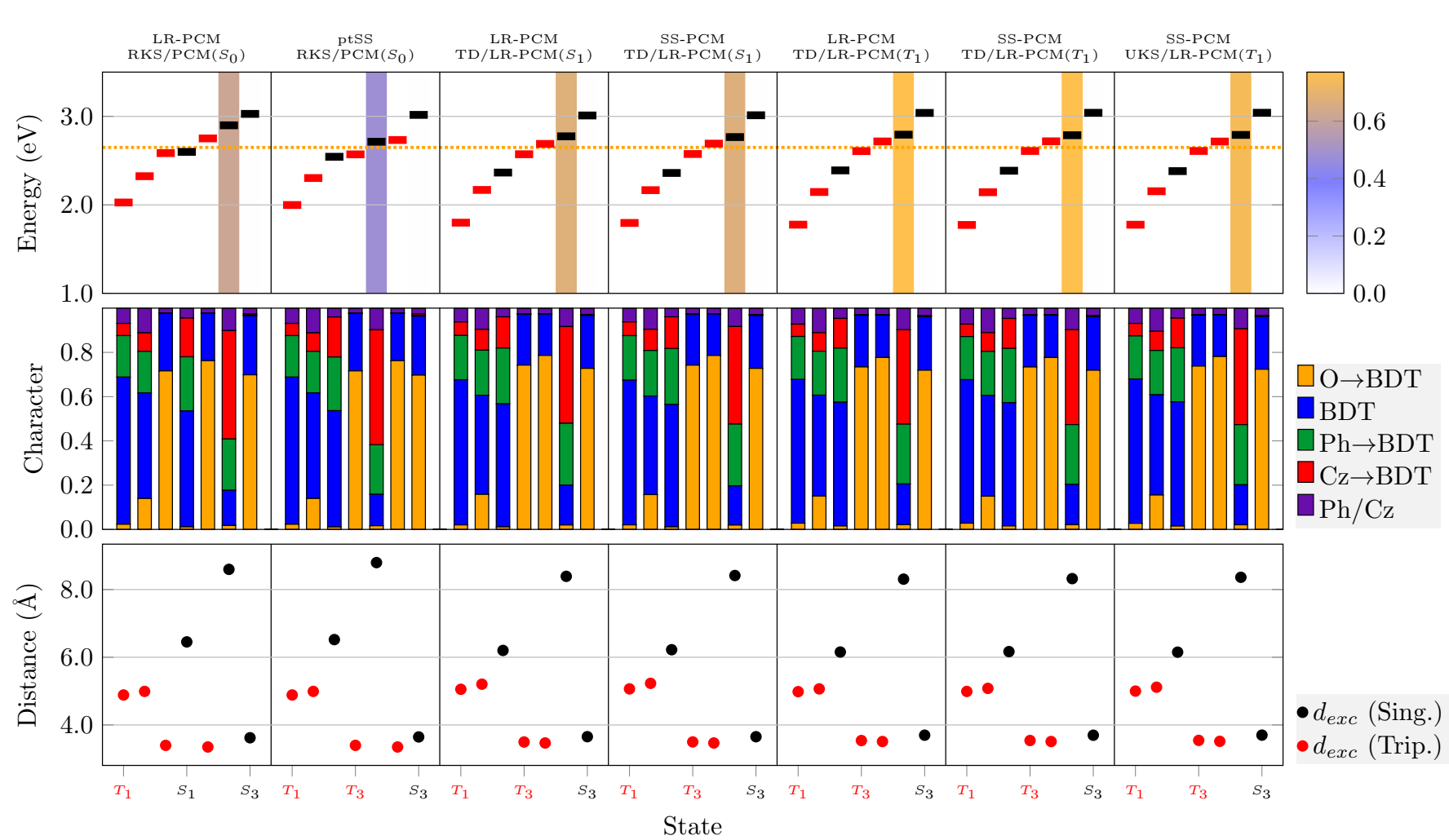

Fig. 8 Analysis of the lowest singlet and triplet states of Cz-BDT at the $S_{0}, T_{1}$ and $S_{1}$ minimum geometries: Adiabatic energies and oscillator strengths (top), excited-state characters (middle), and charge transfer measures ( $d_{\text {exc }}, d_{h e}$, bottom). The excited-state solvation model and method for computing the geometry are given in the top two lines, always in connection with the $\omega$ PBEh/def2-SVP level of theory.

reduced when compared to the ground state geometry (6.52 $\mathrm{A})$. We can understand this in the sense that the planarisation of the Ph-BDT-Ph unit, as represented by the $\theta_{2} / \theta_{2}^{\prime}$ values in Table 1 . provides the basis for an extended locally excited state. The state retains the formal gerade symmetry present at the $S_{0}$ geometry and, therefore, has vanishing oscillator strength.

A locally excited and optically dark $S_{1}$ minimum certainly runs counter to the initial design strategy of using a D- $\pi$-A- $\pi$-D system and may well explain the poor performance of Cz-BDT in TADF applications. To further test this hypothesis, we have spent some effort in searching for a different local minimum on the $S_{1}$ energy surface that might have enhanced CT character considering two strategies: (i) pre-optimisation with the PBE functional before starting the $\omega \mathrm{PBEh}$ optimisation and (ii) optimisation of
$S_{2}$. Neither of these strategies led to the location of an $S_{1}$ minimum with enhanced CT character and we conclude that, indeed, the excitation localises on the Ph-BDT-Ph part upon excited-state relaxation. Furthermore, we find that inclusion of state-specific solvation using the toluene solvent only has a minor overall effect of the energies. All energy shifts are below $0.01 \mathrm{eV}$ and the state characters and exciton sizes virtually unaffected suggesting that the conclusions made are robust with respect to the specifics of the solvation model used.

Proceeding to $T_{1}$, we compare data using three different methods: TDDFT/LR-PCM and TDDFT/SS-PCM at the TDDFT/LRPCM geometry, as well as TDDFT/SS-PCM at the UKS/PCM $T_{1}$ geometry. In all cases we find that optimisation of $T_{1}$ leads to a slight stabilisation of $T_{1}$ obtaining an adiabatic $T_{1}$ energy of 
$\sim 1.8 \mathrm{eV}$ retaining local character with an exciton size of around $5 \AA$. Furthermore, we find that energies and state characters between the $S_{1}$ and $T_{1}$ optimised geometries are very similar, which is consistent with the fact that also their geometric parameters are very similar (Table 3).

Results for geometry optimisiations of the individual states for Cz-AQ are presented in (Figure 9). At the $S_{0}$ minimum, we find similar state characters as in vacuo (Figure S2, centre) only that, again, the $\pi \pi^{*}$ states are somewhat reduced in energy altering the overall state ordering. Once again, we find excellent agreement between the bright $S_{2}$ state obtained at $2.97 \mathrm{eV}$ using the ptSS scheme and the experimental absorption maximum of $2.91 \mathrm{eV}$. Unlike Cz-BDT, there are notable differences in the energies and state characters at the individual geometries, which also agrees well with the fact that the geometries obtained (see Table 3) are quite different to each other. Crucially, we find for the TDDFT optimised $S_{1}$ geometry that the $S_{1}$ state becomes bright and has a significant amount of charge transfer character (red) along with an enhanced exciton size of $7.99 \AA$ (SS-PCM). We propose to interpret this phenomenon in the context of excited-state symmetry breaking: ${ }^{984}$ The $S_{1}$ state is Laporte forbidden at the ground state geometry due to its approximate inversion symmetry but this restriction is lifted once the symmetry is broken in the excited state as one half of the molecule planarises (see Table 3). To represent the symmetry breaking, we use the linear electron-hole separation $\left(d_{\text {he }}\right.$, Eq. (7)). This value vanishes for symmetric charge transfer systems but approaches the value of $d_{e x c}$ in the idealised case where charge is transferred from one donor to one acceptor, both represented as point charges. ${ }^{[22}$ As expected, $d_{h e}$ is zero for the symmetric $S_{0}$-optimised geometries. In the case of $S_{1}$, by contrast, we find that for all the $\pi \pi^{*}$ states $d_{h e}$ differs strongly from zero reaching half of $d_{e x c}$ or more. This highlights that symmetry breaking of the geometry does indeed also localise the excited states.

The $T_{1}$ state at the $T_{1}$ geometry has enhanced local character (blue) and a clearly reduced exciton size $(<6 \AA$ ) when compared to $S_{1}$ at the $S_{1}$ geometry considering all three levels of theory considered. Nonetheless, pronounced symmetry-breaking occurs, which is reflected by the brightness of the $S_{1}$ state and the nonvanishing $d_{h e}$ values.

Once again, we find that all solvation schemes afford similar descriptions of the state energies and characters, with the exception of the $T_{4}$ state at the $S_{1}$ geometry, which is swapped in order with the almost degenerate $S_{1}$ state. The small influence of the LR- and SS- solvation schemes can be understood by the fact that the influence of non-polar solvent like toluene on the excited state energies and characters is small; more polar solvents are expected to lower the energy of charge transfer states and reduce $\Delta E_{S T}$ under the SS-PCM solvation scheme.

Reviewing Figures 8 and 9 , we find that both molecules retain significant adiabatic singlet-triplet gaps, even when solvation effects are accounted for. Thus, the singlet-triplet gap does not serve as a suitable figure of merit to differentiate between the two molecules. On the other hand, a clear difference is observed in terms of excited-state character of the optimised $S_{1}$ state. This state becomes a dark locally excited state for Cz-BDT whereas it becomes a bright symmetry-broken CT state for Cz-AQ. The enhanced optical brightness is certainly beneficial for luminescence while also enhanced CT agrees with the underlying design strategy. Both phenomena explain the enhanced performance of $\mathbf{C z}$ AQ. The difference in state character is reflected by the more planar structure of Cz-BDT in its ground and excited states when compared to Cz-AQ. This planar structure along with shorter bond lengths is an indication of enhanced conjugation between $\mathbf{B D T}$ and $\mathbf{P h}$ when compared to $\mathbf{A Q}$ and $\mathbf{P h}$. The difference can, in part, be understood by the reduced steric repulsion between BDT and $\mathbf{P h}$ but electronic effects most probably also play a role as $\mathbf{C z - A Q}$ is, indeed, able to planarise as seen for the $T_{1}$ minimum.

\subsection{Outlook - Cz-BDT-SO2 and Cz-BDF}

Having provided a detailed analysis of Cz-BDT and Cz-AQ, we will now proceed to two additional molecules, alluded to briefly in the introduction and shown in Fig. 1. (i) Cz-BDT-SO ${ }_{2}$ containing an oxidised derivative of the BDT acceptor, with two oxygen atoms bound to each sulfur atom; and (ii) Cz-BDF, the benzodifuran derivative of BDT, where the sulfur atoms are replaced with oxygen atoms. As opposed to Cz-BDT and Cz-AQ, which have been synthesised and well-characterised, these two molecules have not yet been synthesised.

The geometric parameters for Cz-BDT-SO ${ }_{2}$ and Cz-BDF, obtained in analogy to Table 3, are listed in Table 4 Starting with the $S_{0}$ state of Cz-BDT-SO $\mathbf{S}_{2}$, we find that its $\mathbf{C z} / \mathbf{P h}$ torsion angles are almost unaltered when compared to the previous two molecules whereas the core/ $\mathbf{P h}$ torsion angles $\left(\theta_{2} / \theta_{2}^{\prime}\right)$ are significantly reduced indicating that the central $\mathbf{P h}$-core-Ph system is almost planar. Excitation into $T_{1}$ breaks the symmetry for $\mathbf{C z}$ BDT-SO ${ }_{2}$ and one of the angles $\left(\theta_{2}^{\prime}\right)$ planarises even more whereas the other one remains largely unaltered. Interestingly, excitation into $S_{1}$ has the opposite effect and the $\theta_{2} / \theta_{2}^{\prime}$ angles are slightly increased, which is the opposite trend to what was seen for Cz-BDT. Proceeding to $\mathbf{C z}-\mathbf{B D F}$, we find that this molecule stays symmetric and fairly rigid in all states considered possessing a largely planar central $\mathbf{P h}$-core-Ph system. Finally, it is interesting to note that for all four molecules in this work, the bond distances change with the twisting angles. An increase/decrease in $\theta$ leads to an increase/decrease in $d$ showing that the effect of enhanced conjugation produced by a smaller torsion angle also affects the bond distances.

An analysis of the excited states of Cz-BDT-SO ${ }_{2}$ and $\mathbf{C z}-\mathbf{B D F}$ at their $S_{0}, S_{1}$, and $T_{1}$ optimised geometries in solution is presented in Fig. 10 The data shown is analogous to Figs 8 and 9 with the exception that we only present results using the higher-level state-specific solvation models here. Starting with Cz-BDT-SO ${ }_{2}$ shown on the left in Fig. 10 we find that the excitation energies are significantly reduced when compared to Cz-BDT with all seven calculated excited states at or below $2.0 \mathrm{eV}$ in the ptSS scheme at $S_{0}$ geometry. The two low lying triplet states are predominantly locally excited, with only slightly enhanced CT character when compared to Cz-BDT. The $S_{1}$ is found to be a CT state with predominant $\mathrm{Cz} \rightarrow$ core contributions located at $1.73 \mathrm{eV}$ and, as opposed to Cz-BDT, $S_{1}$ is bright with an oscillator strength of 


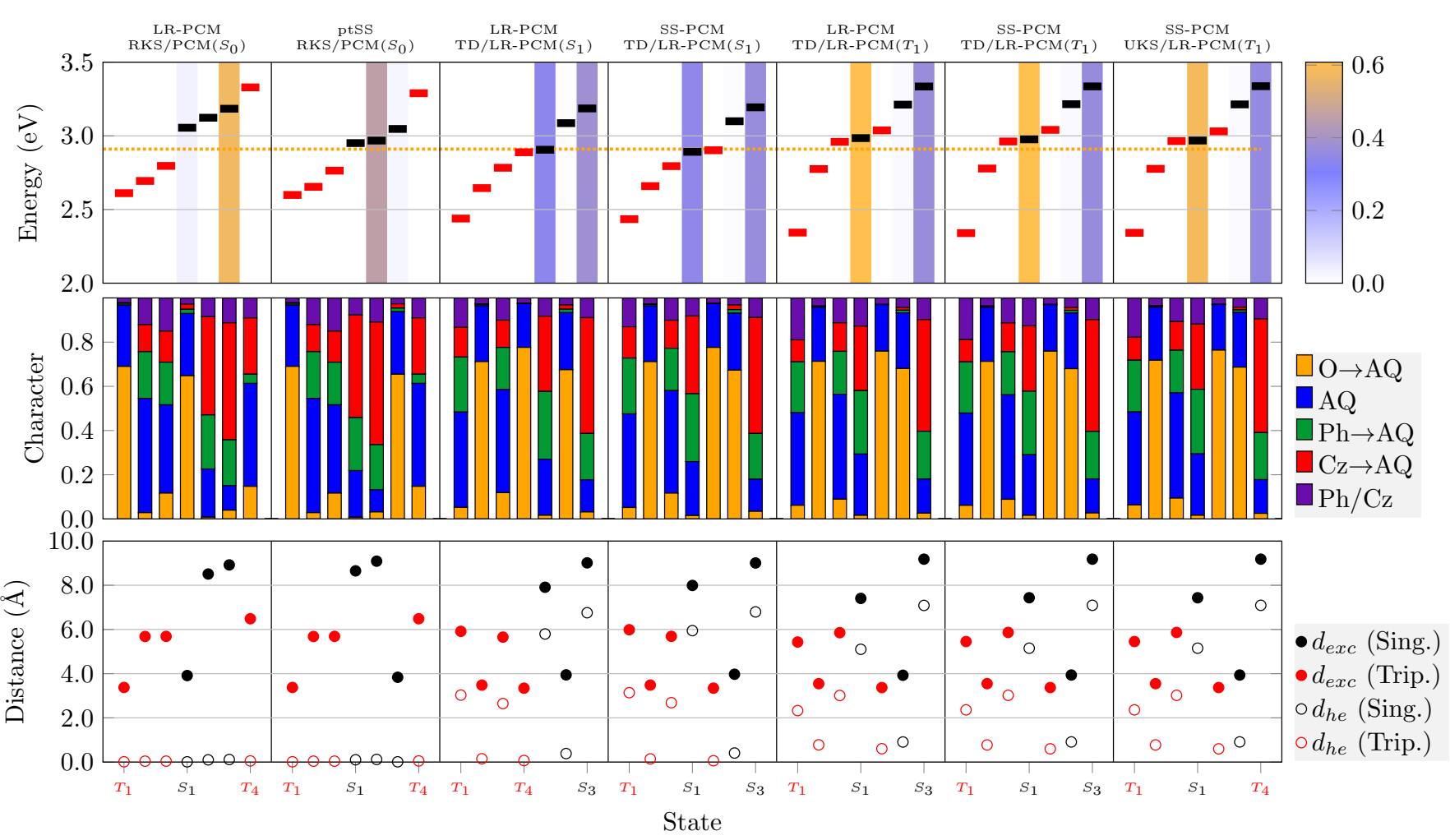

Fig. 9 Analysis of the lowest singlet and triplet states of $\mathrm{Cz}-\mathrm{AQ}$ at the $S_{0}, T_{1}$ and $S_{1}$ minimum geometries: Adiabatic energies and oscillator strengths (top), excited-state characters (middle), and charge transfer measures $\left(d_{e x c}, d_{h e}\right.$, bottom). The excited-state solvation model and method for computing the geometry are given in the top two lines, always in connection with the $\omega$ PBEh/def2-SVP level of theory.

Table 4 Geometric parameters for $\mathrm{Cz}-\mathrm{BDT}-\mathrm{SO}_{2}$ and $\mathrm{Cz}$-BDF obtained at the $\omega$ PBEh/def2-SVP level of theory with a toluene PCM solvent model. Only symmetry-unique values are given.

\begin{tabular}{ccccccc}
\hline Molecule & Geometry & Method & $\theta_{1} / \theta_{1}^{\prime}$ & $d_{1} / d_{1}^{\prime}$ & $\theta_{2} / \theta_{2}^{\prime}$ & $d_{2} / d_{2}^{\prime}$ \\
\hline Cz-BDT-SO & & & & & & \\
& $S_{0}$ min & RKS & $-49.9 /-49.8$ & 1.404 & $6.9 / 6.6$ & 1.499 \\
& $S_{1}$ min & TDDFT & $-55.1 /-48.4$ & $1.412 / 1.401$ & $11.6 / 9.4$ & $1.458 / 1.450$ \\
& $T_{1}$ min & TDDFT & $-50.9 /-43.3$ & $1.407 / 1.389$ & $7.1 / 2.4$ & $1.451 / 1.421$ \\
Cz-BDF & & & & & & \\
& $S_{0}$ min & RKS & -54.9 & 1.412 & -1.9 & 1.457 \\
& $S_{1}$ min & TDDFT & -48.6 & 1.403 & 2.9 & 1.442 \\
& $T_{1}$ min & TDDFT & -49.4 & 1.404 & 3.1 & 1.440 \\
\hline
\end{tabular}

0.68. $S_{2}$ possesses similar character as $S_{1}$ but is optically dark for symmetry reasons. $S_{3}$ is found to be of almost pure $\mathbf{C z} \rightarrow$ core character (red). Two triplets with mixed CT character $\left(T_{3}\right)$ and $\mathrm{n} \pi^{*}$ character $\left(T_{4}\right)$ follow.

Optimisation of Cz-BDT-SO $\mathbf{S O}_{2}$ in the $S_{1}$ state only has a negligible effect on energies and characters of the first four excited states. Up to $S_{2}$, the only notable change is some mixing and symmetry breaking between $S_{1}$ and $S_{2}$ meaning that both states obtain some non-vanishing oscillator strength, their amount of CT character is slightly altered, and their $d_{h e}$ values deviate from zero. Importantly, the $S_{1}$ remains bright at the $S_{1}$ geometry meaning that emission from this state is favoured. For the higher excited states some reordering is observed, e.g., the $n \pi^{*}$-state disappears and all states obtain some partial CT character. Optimisation of $T_{1}$ further stabilises the $T_{1}$ energy to $1.06 \mathrm{eV}$, thus, yielding an adiabatic $\Delta E_{S T}$ of $0.67 \mathrm{eV}$. Optimisation of $T_{1}$ also slightly re- duces the CT character of this state, as seen by the exciton size, which is reduced from $5.64 \AA$ at the $S_{0}$ geometry to $5.35 \AA$. At the $T_{1}$ geometry, also the other states obtain reduced CT character as seen by reduced exciton sizes and red $\mathbf{C z} \rightarrow$ core bars.

The excited states of Cz-BDF (Fig. 10, right) bear resemblance to those of Cz-BDT (Fig. 8). At the $S_{0}$ geometry two locally excited triplet states are found before $S_{1}$, which is also locally excited and dark similarly to Cz-BDT and Cz-AQ. $S_{2}$ is found to be a bright CT state at an excitation energy of $2.73 \mathrm{eV}$, which is almost equivalent to Cz-BDT. In addition, two triplet $\mathrm{n} \pi^{*}$ states $\left(T_{3}\right.$ and $T_{4}$ ) and another singlet CT state $\left(S_{3}\right)$ is found. Optimisation of $S_{1}$ slightly lowers its energy (from $2.47 \mathrm{eV}$ to $2.30 \mathrm{eV}$ ) while retaining its local character. The other states remain largely unaffected when compared between the $S_{0}$ and $S_{1}$ geometries. However, it is noteworthy that the gap between $S_{1}$ and $S_{2}$ becomes $0.51 \mathrm{eV}$ after optimisation. A dark $S_{1}$ state along with a large gap to $S_{2}$ 


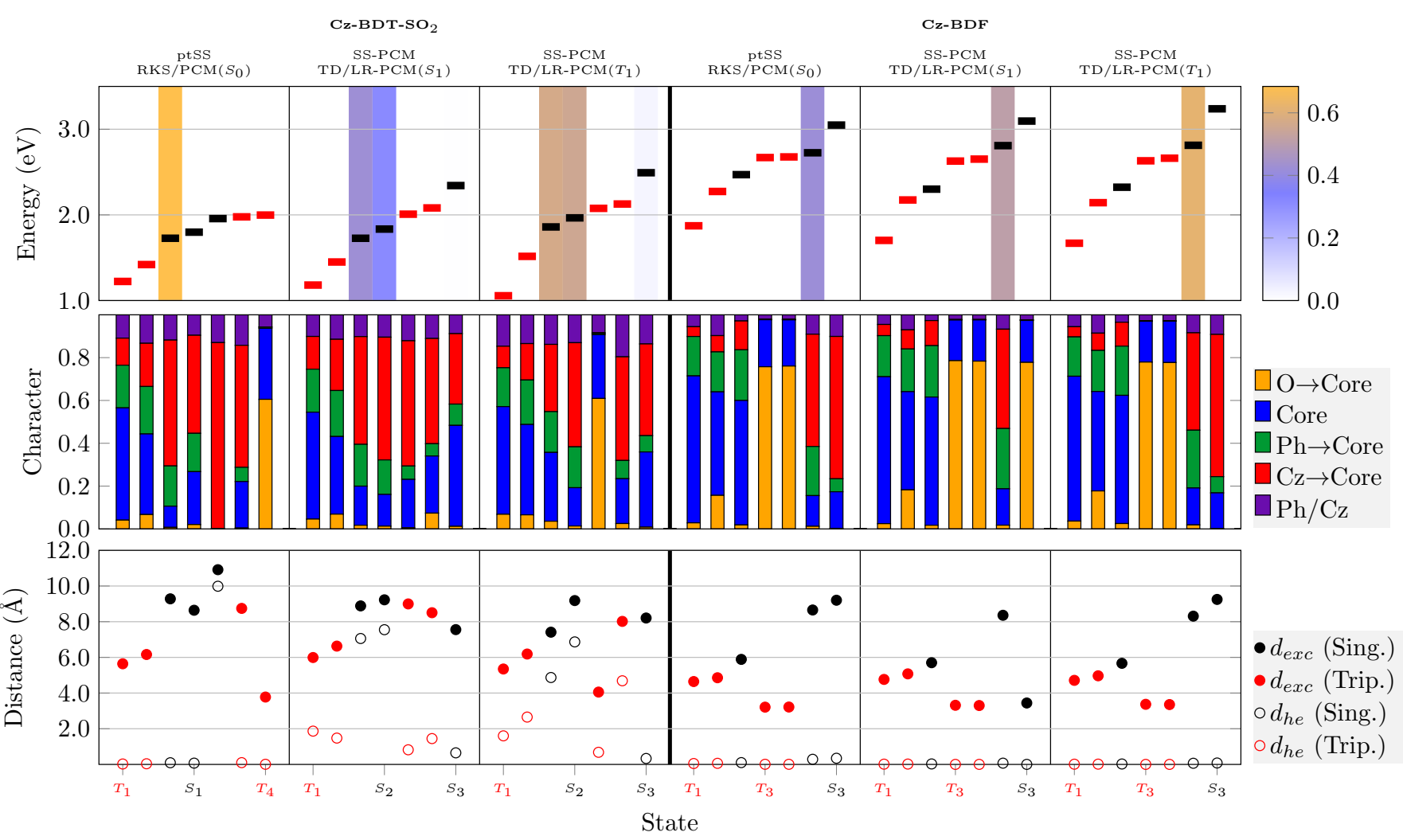

Fig. 10 Analysis of the lowest singlet and triplet states of $\mathrm{Cz}-\mathrm{BDT}-\mathrm{SO}_{2}$ (left) and $\mathrm{Cz}-\mathrm{BDF}$ (right) at the $S_{0}, T_{1}$ and $S_{1}$ minimum geometries: Adiabatic energies and oscillator strengths (top), excited-state characters (middle), and charge transfer measures $\left(d_{e x c}, d_{h e}\right.$, bottom).

indicates that any coupling between the states is expected to be small and Cz-BDF is expected to be at most weakly emissive, similarly to Cz-BDT. Optimisation of $T_{1}$ slightly stabilises the energy of this state inducing an adiabatic singlet-triplet gap of $0.63 \mathrm{eV}$. Otherwise, the states remain largely unaffected.

\section{Discussion}

Before concluding, we want to summarise and discuss the results, presented above, in the context of three specific issues: (i) the overall photophysics of the molecules studied, (ii) general differences in singlet and triplet state wavefunctions and their relevance in terms of photophysics and computational modelling, and (iii) further methodological aspects.

\subsection{Summary of the photophysics}

A summary of the key photophysical data for the four molecules is presented in Table 5 Here, we consider our highest level results, i.e., absorption energies at the TDDFT/ptSS level and emission energies using TDDFT/SS-PCM all with the $\omega$ PBEh functional. We, first, observe that the protocol used works well for reproducing the absorption maxima for Cz-BDT and Cz-AQ whereas the Stokes shifts leading to strongly red-shifted emission are somewhat underestimated. Within the four molecules considered, the absorption energies are arranged as Cz-AQ $>\mathbf{C z}-\mathbf{B D F} \approx \mathbf{C z}-\mathbf{B D T}$ $\gg$ Cz-BDT-SO $_{2}$ reflecting the different acceptor properties of the cores used.

Notably, all four molecules studied have adiabatic singlet- triplet above $0.5 \mathrm{eV}$, which, at first sight, does not seem to make them optimal TADF emitters. Reviewing Fig. 9. we can speculate that despite this energy gap Cz-AQ profits from a high density of states connecting the $T_{1}$ and $S_{1}$ states (see also Ref. 8). In addition, the presence of $n \pi^{*}$ states in this area is expected to mediate SOC efficiently. Finally, symmetry breaking in the excited state produces a strongly emissive CT state $\left(f_{e m}=0.341\right)$ localised on one branch. The presence of a number of different excited states with varying properties, as found here, is consistent with the rich photophysics measured experimentally for a related D-AQ-D molecules. $85 \mid 86$

Viewing Tab. 5, we find the main difference between Cz-AQ and Cz-BDT to be in the oscillator strength for emission $\left(\mathrm{f}_{e m}\right)$, which vanishes for Cz-BDT. We attribute this difference to the absence of a symmetry breaking CT state for Cz-BDT, which, in turn, is caused by a localisation of the excited state on the central Ph-BDT-Ph part mediated by enhanced planarisation.

Considering Tab. 5, we find that Cz-BDT-SO ${ }_{2}$ shows similar characteristics to $\mathbf{C z}-\mathbf{A Q}$ only that the absorption and emission energies are strongly red-shifted and that the singlet-triplet gap is slightly raised. Cz-BDT-SO ${ }_{2}$ may, therefore, be considered a TADF candidate assuming that its $\Delta E_{S T}$ is still sufficiently low. Cz-BDF, on the other hand, has very similar characteristics to Cz-BDT in all of the values considered in Tab.5 It is, thus, expected to exhibit a low photoluminescence quantum yield similarly to Cz-BDT. 
Table 5 Summary of the photophysical data for the four molecules studied.

\begin{tabular}{llllllll}
\hline & $E_{a b s, \max }(\mathrm{eV})$ & $\mathrm{f}_{a b s}$ & $E_{\text {em,max }}(\mathrm{eV})$ & $\mathrm{f}_{e m}$ & $\Phi$ & $\Delta E_{S T, \text { vertical }}(\mathrm{eV})$ & $\Delta E_{S T, \text { adiabatic }}(\mathrm{eV})$ \\
\hline Cz-BDT & 2.71 & 0.478 & 2.19 & 0.000 & - & 0.54 & 0.59 \\
Cz-BDT (exp.) $)^{\mathrm{a}}$ & 2.65 & - & 1.87 & - & 0.095 & - & - \\
Cz-AQ & 2.97 & 0.453 & 2.65 & 0.341 & - & 0.35 & 0.55 \\
Cz-AQ (exp.) ${ }^{\mathrm{b}}$ & 2.91 & 0.19 & 2.22 & - & 0.60 & - & - \\
Cz-BDT-SO $_{2}$ & 1.73 & 0.684 & 1.59 & 0.423 & - & 0.51 & 0.67 \\
Cz-BDF & 2.73 & 0.419 & 2.10 & 0.000 & - & 0.60 & 0.63 \\
\hline
\end{tabular}

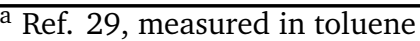

${ }^{\mathrm{b}}$ Ref. 4, measured in toluene

\subsection{Differences between singlet and triplet states}

A striking observation made in the above plots relates to the differences in excited state character found between singlet and triplet states. It is tempting to think of singlet and triplet excited states as involving the same orbital transitions, only differing in their spin coupling. However, the above results show that this picture is oversimplified for realistic push-pull systems. Specifically, we find that low energy triplet states have enhanced local character whereas CT is enhanced for the singlets. This is reflected in all the bar graphs shown in the centre panels of Figs 8 to 10 with enhanced blue and orange bars for the triplets and more red/green for the triplets. The difference is even more apparent in terms of the exciton sizes (bottom panels of Figs 8 to 10 ) showing that the triplet states (red dots) are mostly below $\approx 6 \AA$ whereas, except for $\mathrm{n} \pi^{*}$ states, the singlets (black dots) are well above this value.

Differences between singlet and triplet states can be understood by considering the different contributing energy terms. 15728 Singlet and triplet states are both affected by an attractive Coulomb interaction favouring locally excited states while singlets are also affected by a repulsive exchange interaction favouring CT states. 7 The discussion for triplet energies is a bit more ambiguous and depends on the reference chosen. If one considers the Kohn-Sham orbital energies as reference, then one finds that triplets are independent of any exchange contribution. ${ }^{7}$ However, if one further considers that the LUMO energy is lowered by the exchange interaction between HOMO and LUMO, then one finds that triplet energies are indeed stabilised by this exchange interaction. ${ }^{5}$ Independently of this discussion one finds that low-energy triplets should be more localised, which is well represented by the data shown. Furthermore, the above plots highlight the differences between singlet and triplet states, which cannot be explained by simply reordering the states without also mixing them. Clearly, there is no one-to-one correspondence between the singlet and triplet states.

These differences are important for at least three reasons. First, it should be understood that the photophysics of these molecules cannot be understood in terms of a simplified picture containing only two or three MOs but that a number of terms ultimately influence the final energies of the states. In this context, it has been pointed out, before, that the idealised picture where singlets and triplets differ by twice the exchange energy would only hold if their wavefunctions were the same except for the spin coupling. 87 Discussions of how to go beyond this and understand singlet- triplet gaps within and beyond the orbital picture are provided in Refs 728 . Second, the discussion shows that the available space for molecular design is larger than one would anticipate viewing only two or three MOs. Indeed, there are a large number of terms that can potentially be fine-tuned to optimise the overall photophysics. Third, differences in the wavefunctions of singlet and triplet states explain why singlets and triplets differ strongly in their computational description using, e.g., TDDFT 6488 or the Bethe-Salpeter equation. ${ }^{89}$ Being aware of these differences may help in the development of computational methods that provide a balanced and accurate description of singlets and triplets.

\subsection{Methodological aspects}

Reviewing the computations within this manuscript we found that the density functional used, and in particular the amount of nonlocal HFX, plays a critical role. As shown in Fig. 6 increasing the amount of HFX has four critical consequences: (i) raising the overall excitation energies, (ii) widening $\Delta E_{S T}$, (iii) reducing CT character, and (iv) increasing oscillator strengths. This illustrates how a change in density functional does not only shift excitation energies but potentially affects many different aspects of the predicted photophysics.

Furthermore, we found that geometry optimisation in the excited state was critical for two individual effects. First, it allowed for adjustments in the torsion angles altering delocalisation and CT for the individual states. Second, it was seen as the basis for excited state symmetry breaking yielding a strongly emissive state for Cz-AQ whereas for Cz-BDT the $S_{1}$ state remained dark at its symmetric minimum.

Solvation effects were considered using three different approaches: the LR-PCM and SS-PCM methods in connection with TDDFT as well as ground-state PCM in connection with UKS. It was found that solvation produced a slight energetic stabilisation of CT states. However, differences between these solvation models were comparatively small suggesting that the choice of solvation model is not as critical as the choice of density functional and an appropriate treatment of geometry relaxation, at least for weakly polar solvents.

\section{Conclusions}

Within this work, we have presented a detailed study of four closely related D- $\pi$-A- $\pi$-D molecules: the effective TADF chromophore Cz-AQ, its close analogue Cz-BDT, which undergoes 
red-shifted TADF albeit with a much lower quantum yield, and two molecules not yet synthesised Cz-BDT-SO $\mathbf{S}_{2}$ and Cz-BDF. After presenting the main structural parameters, we have presented a detailed evaluation of computational methods benchmarking five density functionals to experimental absorption data and the high-level $a b$ initio computational reference ri-ADC(2). This highlighted that not only the energies but also the overall wavefunctions depend heavily on the functional used and particularly the amount of Hartree-Fock exchange.

Using the $\omega$ PBEh functional, which was shown to represent energies and wavefunctions accurately, we have studied excitedstate minima in solution highlighting the importance of planarisation and excited-state symmetry breaking leading to markedly different photophysics between the molecules despite their similar molecular structures. The adiabatic singlet-triplet gaps of Cz-AQ and Cz-BDT were found to be very similar not providing a suitable figure of merit to differentiate between the two molecules. Conversely, we have related the strong TADF activity of Cz-AQ to the existence of a strongly emissive symmetry broken $S_{1}$ minimum with CT character whereas Cz-BDT formed a dark locally excited $S_{1}$ minimum. Moreover, Cz-AQ was characterised by a dense set of states of different character connecting the $T_{1}$ and $S_{1}$ states providing a pathway between them despite their comparatively large gap.

In a more general sense, we show that a detailed analysis of excited-state wavefunctions can provide detailed insight into the photophysics of push-pull systems compared to a simple analysis of energies and frontier orbitals. General differences between singlet and triplet states have been outlined with triplets being more compact and local whereas enhanced CT was found for singlets. We believe that the presented protocol will be valuable for studying various push-pull systems in the future providing detailed insight into the properties of existing chromophores and providing new design ideas for the future.

\section{Conflicts of interest}

There are no conflicts to declare.

\section{Acknowledgements}

We acknowledge the Lovelace high-performance computing cluster at Loughborough University. PK acknowledges Loughborough University for a university studentship.

\section{References}

1 F. B. Dias, T. J. Penfold and A. P. Monkman, Methods Appl. Fluoresc., 2017, 5, 012001.

2 Z. Yang, Z. Mao, Z. Xie, Y. Zhang, S. Liu, J. Zhao, J. Xu, Z. Chi and M. P. Aldred, Chem. Soc. Rev., 2017, 46, 915-1016.

3 M. Godumala, S. Choi, M. J. Cho and D. H. Choi, J. Mater. Chem. C, 2019, 7, 2172-2198.

4 Q. Zhang, H. Kuwabara, W. J. Potscavage, S. Huang, Y. Hatae, T. Shibata and C. Adachi, J. Am. Chem. Soc., 2014, 136, 18070-18081.

5 X. K. Chen, D. Kim and J. L. Brédas, Acc. Chem. Res., 2018, 51, 2215-2224.
6 J. M. Mewes, Phys. Chem. Chem. Phys., 2018, 20, 12454 12469.

7 P. Kimber and F. Plasser, Phys. Chem. Chem. Phys., 2020, 22, 6058-6080.

8 J. Eng, J. Hagon and T. J. Penfold, J. Mater. Chem. C, 2019, 7, 12942-12952.

9 B. Dereka, D. Svechkarev, A. Rosspeintner, A. Aster, M. Lunzer, R. Liska, A. M. Mohs and E. Vauthey, Nat. Commun., 2020, 11, $1-11$.

10 A. Dreuw, J. L. Weisman and M. Head-Gordon, J. Chem. Phys., 2003, 119, 2943-2946.

11 S. A. Mewes, F. Plasser, A. Krylov and A. Dreuw, J. Chem. Theory Comput., 2018, 14, 710-725.

12 S. Huang, Q. Zhang, Y. Shiota, T. Nakagawa, K. Kuwabara, K. Yoshizawa and C. Adachi, J. Chem. Theory Comput., 2013, 9, 3872-3877.

13 J. Shee and M. Head-Gordon, J. Chem. Theory Comput., 2020, 16, 6244-6255.

14 R. Improta, V. Barone, G. Scalmani and M. J. Frisch, J. Chem. Phys., 2006, 125, 054103.

15 J. M. Mewes, Z. Q. You, M. Wormit, T. Kriesche, J. M. Herbert and A. Dreuw, J. Phys. Chem. A, 2015, 119, 5446-5464.

16 J. Gibson, A. P. Monkman and T. J. Penfold, ChemPhysChem, 2016, 17, 2956-2961.

17 T. J. Penfold, F. B. Dias and A. P. Monkman, Chem. Commun., 2018, 54, 3926-3935.

18 M. J. G. Peach, P. Benfield, T. Helgaker and D. J. Tozer, J. Chem. Phys., 2008, 128, 044118.

19 C. A. Guido, P. Cortona and C. Adamo, J. Chem. Phys., 2014, 140, 104101.

20 T. Etienne, X. Assfeld and A. Monari, J. Chem. Theor. Comput., 2014, 10, 3896-3905.

21 P. Petelenz and B. Pac, J. Am. Chem. Soc., 2013, 135, 1737917386.

22 F. Plasser, B. Thomitzni, S. A. Bäppler, J. Wenzel, D. R. Rehn, M. Wormit and A. Dreuw, J. Comput. Chem., 2015, 36, 16091620.

23 S. A. Bäppler, F. Plasser, M. Wormit and A. Dreuw, Phys. Rev. A, 2014, 90, 052521.

24 S. A. Mewes, F. Plasser and A. Dreuw, J. Phys. Chem. Lett., 2017, 8, 1205-1210.

25 S. Mai, F. Plasser, J. Dorn, M. Fumanal, C. Daniel and L. González, Coord. Chem. Rev., 2018, 361, 74-97.

26 F. Glöcklhofer, A. Rosspeintner, P. Pasitsuparoad, S. Eder, J. Fröhlich, G. Angulo, E. Vauthey and F. Plasser, Mol. Syst. Des. Eng., 2019, 4, 951-961.

27 Z. Pei, J. Yang, J. Deng, Y. Mao, Q. Wu, Z. Yang, B. Wang, C. M. Aikens, W. Liang and Y. Shao, Phys. Chem. Chem. Phys., 2020, 22, 26852-26864.

28 Z. Pei, Q. Ou, Y. Mao, J. Yang, A. de la Lande, F. Plasser, W. Liang, Z. Shuai and Y. Shao, J. Phys. Chem. Lett., 2021, 12, 2712-2720.

29 S. Montanaro, A. J. Gillett, S. Feldmann, E. W. Evans, 
F. Plasser, R. H. Friend and I. A. Wright, Phys. Chem. Chem. Phys., 2019, 21, 10580-10586.

30 L. Ye, S. Zhang, W. Zhao, H. Yao and J. Hou, Chem. Mater., 2014, 26, 3603-3605.

31 L. Ye, X. Jiao, H. Zhang, S. Li, H. Yao, H. Ade and J. Hou, Macromolecules, 2015, 48, 7156-7163.

32 D. Patra, W. Budiawan, T. Y. Huang, K. H. Wei, P. C. Wang, K. C. Ho, M. Al-Hashimi and C. W. Chu, ACS Appl. Energy Mater., 2018, 1, 3684-3692.

33 G. Huang, H. Jiang, J. Zhang, F. Liu, M. Zhu, H. Tan, Y. Wang, W. Chen, R. Yang and W. Zhu, ACS Appl. Energy Mater., 2018, 1, 1506-1511.

34 T. Nakamura, Macromolecules, 2020, 53, 9836-9846.

35 T. J. Aldrich, W. Zhu, S. Mukherjee, L. J. Richter, E. Gann, D. M. DeLongchamp, A. Facchetti, F. S. Melkonyan and T. J. Marks, Chem. Mater., 2019, 31, 4313-4321.

36 T. J. Aldrich, A. S. Dudnik, N. D. Eastham, E. F. Manley, L. X. Chen, R. P. Chang, F. S. Melkonyan, A. Facchetti and T. J. Marks, Macromolecules, 2018, 51, 9140-9155.

37 K. Kobayashi, C. L. Gajurel, K. Umemoto and Y. Mazaki, Bull. Chem. Soc. Jpn., 1992, 65, 2168-2172.

38 S. Yamaguchi, H. Tatemitsu, Y. Sakata and S. Misumi, Chem. Lett., 1983, 12, 1229-1230.

39 J. H. Kim, J. H. Yun and J. Y. Lee, Adv. Opt. Mater., 2018, 6, 1800255.

40 C. J. Christopherson, D. M. Mayder, J. Poisson, N. R. Paisley, C. M. Tonge and Z. M. Hudson, ACS Appl. Mater. Interfaces, 2020, 12, 20000-20011.

41 D. G. Congrave, B. H. Drummond, P. J. Conaghan, H. Francis, S. T. Jones, C. P. Grey, N. C. Greenham, D. Credgington and H. Bronstein, J. Am. Chem. Soc., 2019, 141, 18390-18394.

42 J. Chen, W. Tao, W. Chen, Y. Xiao, K. Wang, C. Cao, J. Yu, S. Li, F. Geng, C. Adachi, C. Lee and X. Zhang, Angew. Chemie Int. Ed., 2019, 58, 14660-14665.

43 X. Gong, P. Li, Y. Huang, C. Wang, C. Lu, W. Lee, C. Zhong, Z. Chen, W. Ning, C. Wu, S. Gong and C. Yang, Adv. Funct. Mater., 2020, 30, 1908839.

44 E. Avellanal-Zaballa, A. Prieto-Castaneda, F. Garcia-Garrido, A. R. Agarrabeitia, E. Rebollar, J. Banuelos, I. Garcia-Moreno and M. J. Ortiz, Chem. - A Eur. J., 2020, 26, 16080-16088.

45 T. Yang, Z. Cheng, Z. Li, J. Liang, Y. Xu, C. Li and Y. Wang, Adv. Funct. Mater., 2020, 30, 2002681.

46 B. Zhao, H. Wang, C. Han, P. Ma, Z. Li, P. Chang and H. Xu, Angew. Chemie Int. Ed., 2020, 59, 19042-19047.

47 P. Kautny, F. Glöcklhofer, T. Kader, J.-M. Mewes, B. Stöger, J. Fröhlich, D. Lumpi and F. Plasser, Phys. Chem. Chem. Phys., 2017, 19, 18055-18067.

48 S. Zhen, S. Wang, S. Li, W. Luo, M. Gao, L. G. Ng, C. C. Goh, A. Qin, Z. Zhao, B. Liu and B. Z. Tang, Adv. Funct. Mater., 2018, 28, 1706945.

49 E. Varathan and V. Subramanian, Phys. Chem. Chem. Phys., 2017, 19, 12002-12012.

50 E. Varathan and A. Patnaik, J. Phys. Chem. A, 2019, 123, 8755-8765.
51 F. Plasser, J. Chem. Phys., 2020, 152, 084108.

52 F. Plasser, M. Wormit and A. Dreuw, J. Chem. Phys., 2014, 141, 024106.

53 F. Plasser and H. Lischka, J. Chem. Theory Comput., 2012, 8, 2777-2789.

54 R. L. Martin, J. Chem. Phys., 2003, 118, 4775-4777.

55 F. Plasser, A. J. Aquino, W. L. Hase and H. Lischka, J. Phys. Chem. A, 2012, 116, 11151-11160.

56 S. Rupp, F. Plasser and V. Krewald, Eur. J. Inorg. Chem., 2020, 2020, 1506-1518.

57 P. A. Sánchez-Murcia, J. J. Nogueira, F. Plasser and L. González, Chem. Sci., 2020, 11, 7685-7693.

58 S. Yang, D. Streater, C. Fiankor, J. Zhang and J. Huang, J. Am. Chem. Soc., 2021, 143, 1061-1068.

59 N. Mardirossian and M. Head-Gordon, Phys. Chem. Chem. Phys., 2014, 16, 9904-9924.

60 F. Weigend and R. Ahlrichs, Phys. Chem. Chem. Phys., 2005, 7, 3297-3305.

61 C. Hättig, Adv. Quantum Chem., 2005, 50, 37-60.

62 A. Schäfer, C. Huber and R. Ahlrichs, J. Chem. Phys., 1994, 100, 5829-5835.

63 S. Hirata and M. Head-Gordon, Chem. Phys. Lett., 1999, 314, 291-299.

64 M. J. Peach, M. J. Williamson and D. J. Tozer, J. Chem. Theory Comput., 2011, 7, 3578-3585.

65 J. P. Perdew, K. Burke and M. Ernzerhof, Phys. Rev. Lett., 1996, 77, 3865-3868.

66 C. Adamo and V. Barone, J. Chem. Phys., 1999, 110, 61586170.

67 M. A. Rohrdanz, K. M. Martins and J. M. Herbert, J. Chem. Phys., 2009, 130, 054112.

68 T. Yanai, D. P. Tew and N. C. Handy, Chem. Phys. Lett., 2004, 393, 51-57.

69 A. D. Becke, J. Chem. Phys., 1993, 98, 5648-5652.

70 C. Lee, W. Yang and R. G. Parr, Phys. Rev. B, 1988, 37, 785789.

71 V. Barone and M. Cossi, J. Phys. Chem. A, 1998, 102, 19952001.

72 M. Cossi, N. Rega, G. Scalmani and V. Barone, J. Comput. Chem., 2003, 24, 669-681.

73 R. Cammi and B. Mennucci, J. Chem. Phys., 1999, 110, $9877-$ 9886.

74 M. Cossi and V. Barone, J. Chem. Phys., 2001, 115, 47084717.

75 Z. Q. You, J. M. Mewes, A. Dreuw and J. M. Herbert, J. Chem. Phys., 2015, 143, 204104.

76 J. M. Mewes, J. M. Herbert and A. Dreuw, Phys. Chem. Chem. Phys., 2017, 19, 1644-1654.

77 Y. Shao, Z. Gan, E. Epifanovsky, A. T. Gilbert, M. Wormit, J. Kussmann, A. W. Lange, A. Behn, J. Deng, X. Feng, D. Ghosh, M. Goldey, P. R. Horn, L. D. Jacobson, I. Kaliman, R. Z. Khaliullin, T. Kuś, A. Landau, J. Liu, E. I. Proynov, Y. M. Rhee, R. M. Richard, M. A. Rohrdanz, R. P. Steele, E. J. Sund- 
strom, H. L. Woodcock, P. M. Zimmerman, D. Zuev, B. Albrecht, E. Alguire, B. Austin, G. J. O. Beran, Y. A. Bernard, E. Berquist, K. Brandhorst, K. B. Bravaya, S. T. Brown, D. Casanova, C.-M. Chang, Y. Chen, S. H. Chien, K. D. Closser, D. L. Crittenden, M. Diedenhofen, R. A. DiStasio, H. Do, A. D. Dutoi, R. G. Edgar, S. Fatehi, L. Fusti-Molnar, A. Ghysels, A. Golubeva-Zadorozhnaya, J. Gomes, M. W. Hanson-Heine, P. H. Harbach, A. W. Hauser, E. G. Hohenstein, Z. C. Holden, T.-C. Jagau, H. Ji, B. Kaduk, K. Khistyaev, J. Kim, J. Kim, R. A. King, P. Klunzinger, D. Kosenkov, T. Kowalczyk, C. M. Krauter, K. U. Lao, A. D. Laurent, K. V. Lawler, S. V. Levchenko, C. Y. Lin, F. Liu, E. Livshits, R. C. Lochan, A. Luenser, P. Manohar, S. F. Manzer, S.-P. Mao, N. Mardirossian, A. V. Marenich, S. A. Maurer, N. J. Mayhall, E. Neuscamman, C. M. Oana, R. Olivares-Amaya, D. P. O’Neill, J. A. Parkhill, T. M. Perrine, R. Peverati, A. Prociuk, D. R. Rehn, E. Rosta, N. J. Russ, S. M. Sharada, S. Sharma, D. W. Small, A. Sodt, T. Stein, D. Stück, Y.-C. Su, A. J. Thom, T. Tsuchimochi, V. Vanovschi, L. Vogt, O. Vydrov, T. Wang, M. A. Watson, J. Wenzel, A. White, C. F. Williams, J. Yang, S. Yeganeh, S. R. Yost, Z.-Q. You, I. Y. Zhang, X. Zhang, Y. Zhao, B. R. Brooks, G. K. Chan, D. M. Chipman, C. J. Cramer, W. A. Goddard, M. S. Gordon, W. J. Hehre, A. Klamt, H. F. Schaefer, M. W. Schmidt, C. D. Sherrill, D. G. Truhlar, A. Warshel, X. Xu, A. Aspuru-Guzik, R. Baer, A. T. Bell, N. A. Besley, J.-D. Chai, A. Dreuw, B. D. Dunietz, T. R. Furlani, S. R. Gwaltney, C.-P. Hsu, Y. Jung, J. Kong, D. S. Lambrecht, W. Liang, C. Ochsenfeld, V. A. Rassolov, L. V. Slipchenko, J. E. Subotnik, T. Van Voorhis, J. M. Herbert, A. I.
Krylov, P. M. Gill and M. Head-Gordon, Mol. Phys., 2015, 113, 184-215.

78 Turbomole V7.2 2017, a development of University of Karlsruhe and Forschungszentrum Karlsruhe GmbH, 19892007, TURBOMOLE GmbH, since 2007; available from http://www.turbomole.com.

79 Supporting research data available: molecular geometries, input/output files of Q-Chem and Turbomole. DOI: 10.17028/rd.lboro.15111870 .

80 D. Kánnár and P. G. Szalay, J. Mol. Model., 2014, 20, 2503.

81 D. J. Tozer, R. D. Amos, N. C. Handy, B. O. Roos and L. Serrano-Andres, Mol. Phys., 1999, 97, 859-868.

82 S. A. Mewes, F. Plasser and A. Dreuw, J. Chem. Phys., 2015, 143, 171101.

83 S. Bai, R. Mansour, L. Stojanović, J. M. Toldo and M. Barbatti, J. Mol. Model., 2020, 26, 1-9.

84 Z. Szakács, F. Glöcklhofer, F. Plasser and E. Vauthey, Phys. Chem. Chem. Phys., 2021, 23, 15150-15158.

85 J. Choi, D.-S. Ahn, K. Y. Oang, D. W. Cho and H. Ihee, J. Phys. Chem. C, 2017, 121, 24317-24323.

86 Z. Kuang, G. He, H. Song, X. Wang, Z. Hu, H. Sun, Y. Wan, Q. Guo and A. Xia, J. Phys. Chem. C, 2018, 122, 3727-3737.

87 D. Kim, J. Phys. Chem. C, 2015, 119, 12690-12697.

88 D. Jacquemin, E. A. Perpète, I. Ciofini and C. Adamo, J. Chem. Theory Comput., 2010, 6, 1532-1537.

89 D. Jacquemin, I. Duchemin, A. Blondel and X. Blase, J. Chem. Theory Comput., 2017, 13, 767-783. 